\title{
Defective FGF signaling causes coloboma formation and disrupts retinal neurogenesis
}

\author{
Shuyi Chen ${ }^{1,2}$, Hua $\mathrm{Li}^{1}$, Karin Gaudenz ${ }^{1}$, Ariel Paulson ${ }^{1}$, Fengli Guo ${ }^{1}$, Rhonda Trimble ${ }^{1}$, Allison Peak ${ }^{1}$, \\ Christopher Seidel $^{1}$, Chuxia Deng ${ }^{3}$, Yasuhide Furuta ${ }^{4}$, Ting Xie ${ }^{1,2}$ \\ ${ }^{I}$ Stowers Institute for Medical Research, 1000 East 50th Street, Kansas City, MO 64110, USA; ${ }^{2}$ Department of Anatomy and Cell \\ Biology, University of Kansas School of Medicine, 3901 Rainbow Blvd, Kansas City, KS 66160, USA; ${ }^{3}$ Genetics of Development \\ and Disease Branch, NIDDK, NIH, 10/9/N105, Bethesda, MD 20892, USA; ${ }^{4}$ Department of Biochemistry and Molecular Biology, \\ University of Texas, M.D. Anderson Cancer Center, Houston, TX 77030, USA
}

The optic fissure (OF) is a transient opening on the ventral side of the developing vertebrate eye that closes before nearly all retinal progenitor cell differentiation has occurred. Failure to close the OF results in coloboma, a congenital disease that is a major cause of childhood blindness. Although human genetic studies and animal models have linked a number of genes to coloboma, the cellular and molecular mechanisms driving the closure of the OF are still largely unclear. In this study, we used Cre-LoxP-mediated conditional removal of fibroblast growth factor (FGF) receptors, $F g f r 1$ and $F g f r 2$, from the developing optic cup (OC) to show that FGF signaling regulates the closing of the OF. Our molecular, cellular and transcriptome analyses of $F g f r 1$ and $F g f r 2$ double conditional knockout OCs suggest that FGF signaling controls the OF closure through modulation of retinal progenitor cell proliferation, fate specification and morphological changes. Furthermore, $F g f r 1$ and $F g f r 2$ double conditional mutant retinal progenitor cells fail to initiate retinal ganglion cell (RGC) genesis. Taken together, our mouse genetic studies reveal that FGF signaling is essential for OF morphogenesis and RGC development.

Keywords: optic fissure; coloboma; FGF signaling; retinal ganglion cells

Cell Research (2013) 23:254-273. doi:10.1038/cr.2012.150; published online 13 November 2012

\section{Introduction}

Epithelial sheet fusion is an essential morphogenetic event during embryogenesis, and it is also one of the most important steps in vertebrate eye morphogenesis. When the optic vesicle (OV) approaches the surface ectoderm, it invaginates to form the double-layered optic cup (OC) structure, which contains an inner neural retina (NR) layer and an outer retinal pigmented epithelium (RPE) layer. The invagination process of the OV is asymmetric, oriented in a somewhat ventral-distal to dorsal-medial direction, which leads to the formation of

\footnotetext{
Correspondence: Shuyi Chen ${ }^{\mathrm{a}}$, Ting Xie $\mathrm{Xi}^{\mathrm{b}}$

${ }^{\text {aTTel: }+1-816-926-4060}$

E-mail: sch@stowers.org

${ }^{\mathrm{b}} \mathrm{Tel}:+1-816-926-4061$

E-mail: tgx@stowers.org

Received 3 May 2012; revised 25 July 2012; accepted 10 September 2012; published online 13 November 2012
}

a groove on the ventral OC called the optic fissure (OF) [1, $2]$. The OF extends from the most distal point of the OC to the proximal end of the optic stalk (OS) [1]. The OF provides a channel for surrounding mesenchymal cells to migrate into the developing eye. The mesenchymal cells later form hyaloid vessels for providing blood supply to the developing eye. The formation of the OF relies on the ventral patterning of the OV and is regulated by BMP7 [2, 3]. As development proceeds, the fusion of the OF makes the ventral retina morphologically identical to the dorsal retina [4]. The fissure closure still leaves one opening at the center of the $\mathrm{OC}$, the optic disc, as the passageway for retinal ganglion cell (RGC) axons and blood vessels [3]. Therefore, the timely closure of the OF is critical for normal eye development and function.

The failure of the fissure closure leads to the formation of a permanent opening on the ventral retina, a condition known as coloboma. It affects 0.41 to 2.6 infants in every 10000 births, occurring in isolation or in association with other developmental syndromes [1]. The 
most common syndrome associated with coloboma is the CHARGE syndrome, caused by a mutation in $\mathrm{CHD7}$, a chromatin remodeling factor that controls neural crest cell differentiation [1], suggesting an important role of periocular mesenchymal cells (PMCs) in regulating the OF closure. Haploinsufficiency of Pax2, a member of the paired box family of transcription factors, is the genetic cause for the human renal-coloboma syndrome, which manifests optic nerve excavation [5, 6]. Homozygous Pax 2 mutant mice develop coloboma, accompanied by the extension of the retinal tissue into the optic stalk [7]. Human genetic studies have linked a number of other genes to coloboma, including PAX6, VSX2 (CHX10), SIX3 and PTCH [1]. However, each of them is only responsible for a very small portion of coloboma patients, and it remains unclear how these mutations contribute to the pathogenesis of coloboma mechanistically. In the past two decades, genetic studies in animal models have identified additional coloboma-causing genes, including transcription factors (Vax1, Vax2 [8, 9], Pitx2 [10] and Bfl [11]), signaling pathway components (Jnk1/2 [12], Rxro, Rary [13], Raldh1, Raldh3 [14], Lrp6 [15] and $F z d 5$ [16]), cell cycle regulators (Phactr4 [17]), and polycomb-complex components (Rybp [18]). These studies suggest that successful OF closure might require precisely controlled retinal cell fate specification, balanced retinal cell proliferation, and proper interactions between retina and surrounding tissues.

The mature retina is composed of six types of neurons and one glial cell type that are well organized into three cellular layers, with RGCs occupying the inner most ganglion cell layer; amacrine, bipolar and horizontal interneurons and Müller glia cells residing in the inner nuclear layer; and rod and cone photoreceptor cells composing the outer nuclear layer [19]. These seven types of retinal cells are generated by common multipotent retinal progenitor cells in a sequential yet overlapping manner. From fish to mammals, the RGC has consistently been shown to be the earliest retinal cell type generated by retinal progenitor cells, followed by cone, horizontal and amacrine cells, while rod, bipolar and Müller cells are generated last [19]. Such ordered appearance of different retinal cell types is determined by an intrinsic property, or competence, of retinal progenitor cells, and is influenced by environmental signals [20]. Studies have shown that the competence of retinal progenitor cells to generate RGCs is determined by a basic helix-loop-helix transcription factor, Math5, which promotes RGC fate by regulating the expression of RGC transcription factors, Brn3b and Islet1 [21]. The duration of Math5 expression, thus the competence of retinal progenitor cells to produce RGCs, is controlled by growth and differentiation factor 11 [22]. In addition, Shh produced by early-born RGCs has been shown to provide feedback signaling to retinal progenitor cells to inhibit further RGC generation [23]. Fully understanding the intrinsic factors and extrinsic signals that control retinal neurogenesis will facilitate the development of therapeutic strategies to treat retinal diseases that affect RGCs, such as glaucoma.

Fibroblast growth factor (FGF) signaling is reiteratively employed by different cell types during embryogenesis and adult homeostasis to regulate cell proliferation, survival, migration or differentiation [24, 25]. Studies in zebrafish, frog and chicken have suggested that FGF signaling regulates the initial eye field formation [26], NR specification [27, 28], retinal axial determination [29], and retinal neuron differentiation [30, 31]. Consistently, retina-specific knockout of $S h p 2$, encoding an adaptor protein that functions downstream of many tyrosine kinase receptors including FGFR and EGFR, causes eye developmental defects [32, 33]. However, in mice, most FGF mutants do not manifest any phenotype in the developing eye, potentially due to functional redundancies of different FGF ligands, as there are 22 FGF ligands in the mouse genome [34]. For example, $F g f 1$ and $F g f 2$ are known to be expressed in the surface ectoderm, and their ectopic administration can sufficiently transform RPE cells into the NR fate in fish, chickens, and mice [27, 28, 35]. Surprisingly, neither single nor double mutant mice for the two ligands show any discernible eye defects [3638]. $F g f 9$ and $F g f 15$ are abundantly expressed in the developing NR; however, mutations of these two ligands cause no or only mild eye defects, respectively $[39,40]$. Knockout mice for $F g f 8$, another ligand expressed in the developing eye, die at E7 [41], preventing the study of its actual role in mouse eye development. Therefore, it remains uncertain what exact roles FGF signaling plays in mammalian eye development.

To overcome the functional redundancies of FGF ligands in the developing mammalian eye, we used a conditional knockout strategy to specifically remove functions of two FGF receptor ( $F g f r$ ) genes, $F g f r l$ and $F g f r 2$, from the developing eye. Among four mouse $F g f r$ genes, Fgfr 3 and Fgfr4 single and double knockout mice show no defects in eye development [42-44]. Fgfrl and Fgfr2 knockout mice die too early to permit the study of their roles in eye development (at E7.5 and E4.5, respectively) [45-47]. Here, we show that retina-specific removal of both $F g f r 1$ and $F g f r 2$ results in coloboma formation and defective RGC development. By carefully analyzing the cellular events of OF closure of the wild type and Fgfrl/ Fgfr 2 double-mutant eyes, we propose that FGF signaling controls OF closure by regulating cell fate specification, morphological changes and proliferation. 


\section{Results}

Defective FGF signaling causes coloboma formation

Although FGF signaling is known to control cell proliferation, morphological changes, and cell fate determination in different systems [24, 25], loss-of-function experiments are needed to determine whether FGF signaling plays any essential roles in mammalian eye development. Because Fgfrl and Fgfr2 are two critical receptors for transducing FGF signals in mice [45-47], we used the Cre-LoxP system to conditionally remove $F g f r 1$ and Fgfr 2 from the developing mouse eye using Six3-Cre. Six3-Cre, in which the Cre gene is driven by the Six 3 promoter, starts its expression in the OC and optic stalk at E9.5 [48]. The floxed alleles for Fgfrl [49] and $F g f r 2$ [50] have been previously shown to inactivate $F g f r 1$ and $F g f r 2$ function, respectively, following Cremediated recombination. Six 3-Cre; Fgfr $1^{f x /+} ; F_{f r} 2^{f x x+}$, Six3-Cre; Fgfr $1^{f x / f x} ; \mathrm{Fgfr}^{f x /+}$ and Six3-Cre; Fgfr $\mathrm{I}^{f x /+}$; $F g f r 2^{f x / f x}$ mice do not show any obvious phenotype on the size and structure of developing eyes at E12.5 and P0 (Supplementary information, Figure S1A-S1C') (collectively referred to as controls hereafter). However, all the retinas of the Six 3-Cre; Fgfr $1^{f x / f x} ; F g f r 2^{f x / f x}$ (referred to as $F g f r$-DCK hereafter) mice have a cleft on the ventral side, which is evident at E12.5 (Supplementary information, Figure S1D) and E13.5 (Figure 1B) but is absent in the control retinas (Supplementary information, Figure S1A-S1C and Figure 1A). The ventral cleft persists to adult stage (Figure 1D and Supplementary information, Figures S1D', S1F and S7) in the mutant eyes. Additionally, the control P0 retina has an optic disc at the center of the retina (Figure 1C and Supplementary information, Figure $\mathrm{S} 1 \mathrm{~A}^{\prime}-\mathrm{S} 1 \mathrm{C}^{\prime}$ ), but the $F g f r-D C K$ mutant $\mathrm{P} 0$ retina completely lacks the optic disc (Figure 1D and Supplementary information, Figure S1D'). Finally, the P15 Fgfr-DCK mutant mouse eyes completely lose their optic nerve (Supplementary information, Figure S1E and S1F). These findings indicate that FGF signaling is important for the closure of the OF and the formation of the optic disc and the optic nerve.

To determine how Fgfrl and $F g f r 2$ are involved in the control of the OF closure, we used in situ hybridization to determine their mRNA expression patterns in both the control and mutant eyes. The Fgfrl probe corresponds to the deleted region of the gene and the $F g f r 2$ probe recognizes the deleted region as well as its surrounding 873 nucleotides. Following the deletion of the floxed region of Fgfr2, which encodes part of the third Ig domain and the transmembrane domain [50], the remaining Fgfr2 transcript can still produce a truncated protein, which can be recognized by a commercial polyclonal antibody against the intracellular domain (Supplementary information, Figure S2B). In the E11.5 eye, in situ hybridization revealed that $F g f r 1$ mRNAs are ubiquitously expressed at high levels in the retinal progenitors (Figure 1E), while $F g f r 2$ mRNAs are expressed in those retinal progenitors at very low levels (Figure 1F). Both Fgfrl and Fgfr 2 mRNAs are abundantly expressed in PMCs outside the OC (Figure 1E and 1F). As expected, Fgfr 1 and Fgfr 2 mRNAs in the PMCs of the Fgfr-DCK mutant OC remain unchanged because Six3-Cre is not expressed in those cells (Figure $1 \mathrm{G}$ and $1 \mathrm{H}$ ). In contrast, $F g f r 1 \mathrm{mRNA}$ levels are drastically reduced in the progenitors of the dorsal, temporal and ventral regions of E11.5 Fgfr-DCK mutant retina (Figure 1G). Surprisingly, Fgfrl mRNA level remains largely unchanged in the retinal progenitors on the nasal region of the mutant retina (Figure $1 \mathrm{G}$ ). It is more surprising that the truncated $F g f r 2$ mRNAs are elevated in the temporal and ventral regions of the $F g f r$ $D C K$ mutant retina (Figure $1 \mathrm{H}$ ). We further examined the protein expression patterns of Fgfr2 in the control and FGFR mutant retinas by immuno-staining. In contrast with low Fgfr2 protein levels in the control retina (Supplementary information, Figure S3A), its expression levels increase in the ventral and temporal regions of the $F g f r-D C K$ mutant retina (Supplementary information, Figure S3C). However, there is no upregulation of Fgfr2 protein in any regions in the Six3-cre; Fgfr $1^{f x / f x}$ mutant retina (Supplementary information, Figure S3B), suggesting that simultaneous inactivation of $F g f r l$ and $F g f r 2$ is required for the compensatory upregulation of $F g f r 2$ transcription. To further investigate the knockout efficiencies of Fgfrl and Fgfr2 in different regions of the developing retina, we performed quantitative PCR (qPCR) on the mRNAs isolated from different quadrants of the control and Fgfr-DCK OCs using primers corresponding to the floxed $F g f r 1$ and $F g f r 2$ regions. As expected, the expression of the floxed regions of both Fgfrl and $F g f r 2$ mRNAs significantly decreases in the temporal and ventral regions of the $F g f r-D C K$ mutant retina, but remains relatively unchanged in the nasal retina (Figure $1 \mathrm{~K})$. To understand why Fgfrl and $F g f r 2$ are not deleted in the nasal region of the retina though Six3-Cre is highly expressed in the nasal region of the wild-type OC, we examined Six3-Cre-mediated deletion efficiency in the mutant OCs using the Z/EG reporter, in which GFP is expressed following the removal of the LoxP-flanked cassette [51]. Consistent with the previously published data [48], GFP is expressed throughout the E11.5 retina in control eyes, though with some degree of mosaicism (Figure 1I). In contrast, the $F g f r-D C K$ mutant retina contains GFP-expressing cells in the dorsal, temporal and ventral regions, but not in the nasal region (Figure 

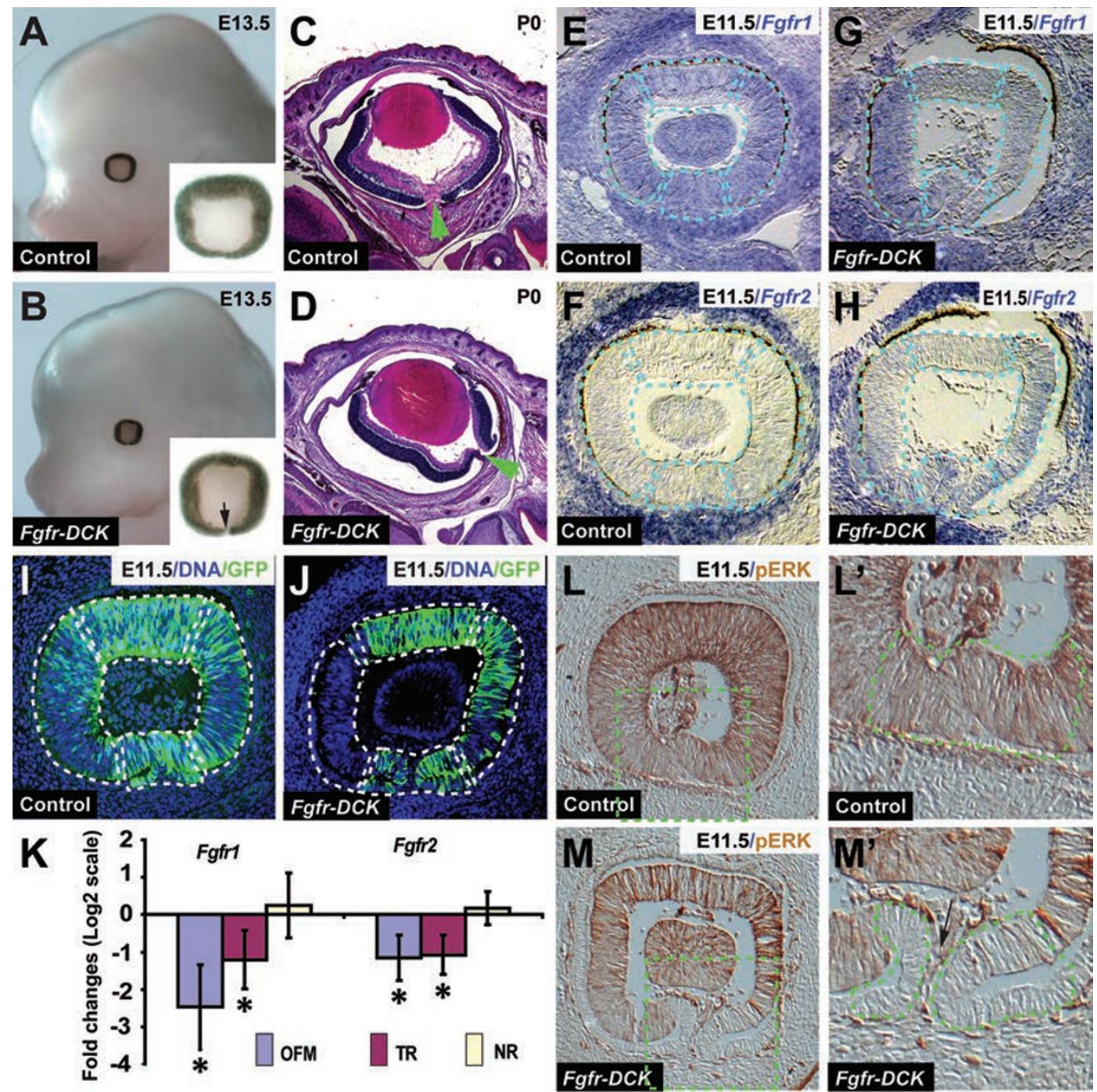

Figure 1 Defective FGF signaling causes coloboma formation. (A, B) E13.5 embryonic heads and eyes. The arrow in B indicates the cleft on the ventral side of the Fgfr-DCK eye. (C, D) Hematoxylin and eosin stained sections of P0 newborn eyes. Green arrowheads in $\mathbf{C}$ points to the optic disc, while in D denotes the unclosed OF. (E, F) Fgfr1 and Fgfr2 mRNAs are expressed in E11.5 retinas at high and low levels, respectively. Four quadrants of the retina are highlighted by dash lines. (G, H) In Fgfr-DCK retinas, Fgfr1 mRNAs are downregulated in dorsal, temporal and OFM quadrants, but remain unchanged in the nasal retina, while the truncated Fgfr2 mRNAs are upregulated in the OFM and temporal regions. (I, J) Six3-cre is expressed throughout the control retina based on the expression of the Z/EG reporter, but its expression in the Fgfr-DCK retina is absent in the nasal region. (K) qPCR results show that the floxed regions of Fgfr1 and Fgfr2 are efficiently deleted by Six3cre in OFM and temporal regions of the Fgfr-DCK retinas, but not in the nasal region. *denotes $P<0.01$. (L, $\mathbf{M}$ ') $p E R K$ is dramatically downregulated in the OFM of the Fgfr-DCK retinas. $\mathbf{L}^{\prime}$ and $\mathbf{M}$ ' represent the squared regions in $\mathbf{L}$ and $\mathbf{M}$ at a higher magnification. Dashed lines in L' and M' highlight OFM.

$1 \mathrm{~J})$. The $Z / E G$ reporter assay can effectively explain why Fgfrl and Fgfr2 are deleted in the dorsal, temporal and ventral regions of the $F g f r-D C K$ retina but not in the nasal region, and further suggests that FGF signaling might have distinct functions in different regions of the retina. Taken together, these results support the idea that functions of Fgfrl and Fgfr 2 can be effectively inactivated in the temporal and ventral regions of the developing retina by Six3-Cre, and also have revealed the existence of the compensatory $\mathrm{Fg} f \mathrm{r} 2$ upregulation in the absence of FGF signaling.

FGF signaling can function through Akt-PI3K, MAPK or both to regulate cell proliferation, cell survival, and cell fate determination $[24,52]$. Phosphorylated Akt 

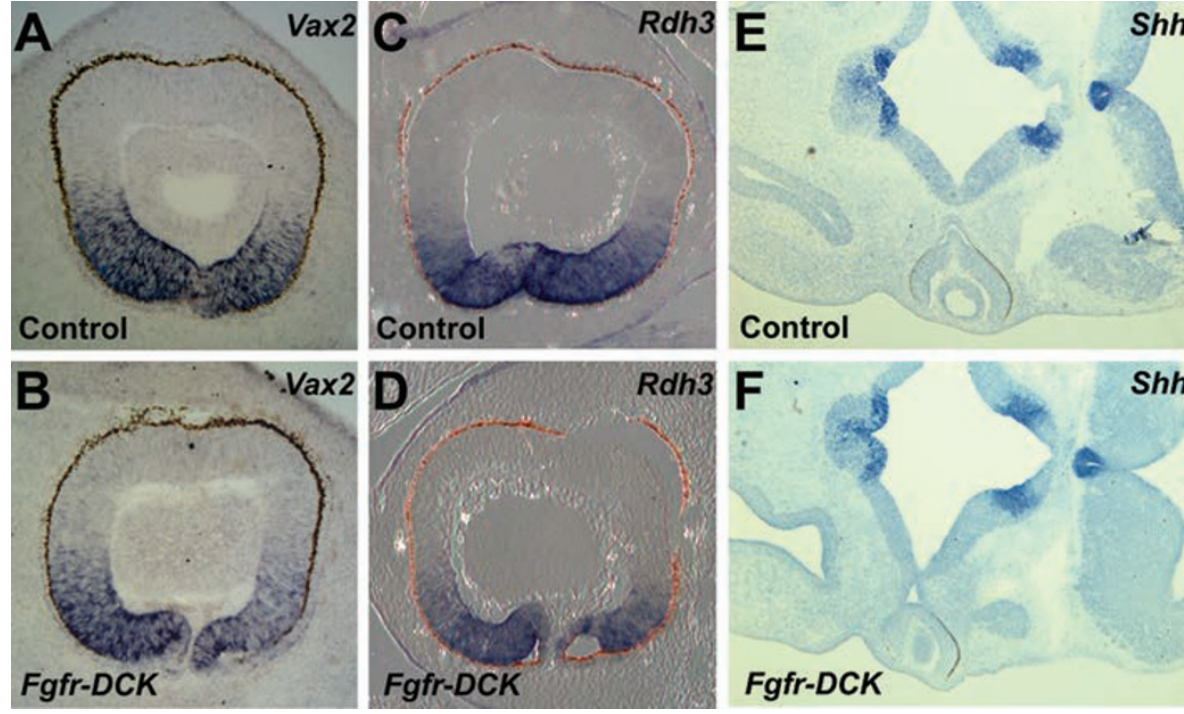

Figure 2 The dorsal-ventral polarity is normally established in the Fgfr-DCK mutant OC. (A, B) Vax2 mRNAs are expressed in the ventral region of the E11.5 control and Fgfr-DCK OCs. (C, D) Raldh3 mRNAs are expressed in the ventral region of the E11.5 control and Fgfr-DCK OCs. (E, F) Shh mRNAs are expressed in the E11.5 forebrain of control and Fgfr-DCK mutant mice.

(pAkt) and MAPK (pERK) are often used to monitor activation of Akt-PI3K and MAPK signaling, respectively. pAKT can only be detected in a few retinal cells of the control retina, and remains unchanged in the Fgfr-DCK mutant retina, indicating it is unlikely that FGF signaling utilizes the Akt-PI3K branch to regulate retinal development (Supplementary information, Figure S4). Interestingly, pERK is highly expressed in all of the retinal progenitor cells, though it appears to exist in a gradient with the highest level on the dorsal side (Figure 1L and 1L'). In the nasal side of $F g f r-D C K$ mutant retinas, pERK is expressed normally as expected due to lack of mutant retinal progenitor cells in the region (Figure 1M). Interestingly, pERK is drastically downregulated in the ventral region but not in dorsal and temporal regions of the mutant retina (Figure $1 \mathrm{M}$ and $\mathrm{M}^{\prime}$ ). These results suggest that $p E R K$ is regulated by FGF signaling in the ventral region of the retina, but not in the dorsal and temporal regions. Because the $\mathrm{OF}$ is located in the ventral region and its closure is affected by FGF signaling, we speculate that FGF-MAPK signaling might play an important role in the ventral region of the retina to control the $\mathrm{OF}$ closure.

FGF signaling is dispensable for general patterning of the dorsal-ventral polarity of the $O C$

Because the OF forms on the ventral side of the eye, perturbation of ventral retinal fate specification has been known to cause coloboma formation. For example, a deletion mutation of Vax2, a ventral retinal fate determinant, leads to coloboma formation [53, 54]. We thus tested if FGF signaling is required for the determination of ventral polarity by examining the expression of $\operatorname{Vax} 2$ and Raldh3, two ventral retina markers [55]. Vax2 and Raldh 3 mRNAs are still expressed in the cells on the ventral side in both the wild-type and Fgfr-DCK mutant eyes, indicating that ventral retina fate is properly specified in Fgfr-DCK mutant retinas (Figure 2A-2D). These results suggest that FGF signaling controls the closure of the OF likely by regulating the behavior of progenitors in the ventral region of the $\mathrm{OC}$.

In the Six3-Cre mice, Cre is expressed not only in the retina but also in the developing ventral forebrain from around embryonic day 9 [48]. Early eye development is regulated by Shh derived from forebrain tissues, which promotes the proximal fate while repressing the distal fate of the OV $[56,57]$. In addition, Shh signaling has been implicated in the induction of Pax 2 and thus the OF closure $[3,11,12]$. To exclude the possibility that the coloboma phenotype in $F g f r$ - DCK mice results from a defect in the production of the midline $S h h$ signal, we examined Shh mRNA expression in the control and FgfrDCK mutant mice. In situ hybridization on E11.5 brain frontal sections shows that $S h h$ is normally expressed in the forebrain midline in $F g f r-D C K$ mutant mice as in the control (Figure 2E-2F). These results suggest that coloboma formation in Fgfr-DCK mutant mice is unlikely caused by defective Shh signal production in the brain. 
However, we do not rule out the possibility that FGF signaling regulates Shh signal transduction in developing retinal progenitors.

FGF signaling maintains $O F$ margin progenitors and controls their subsequent switches into NR and RPE progenitors

As the coloboma phenotype is often accompanied by cell fate determination defects around the fissure area [1], we first investigated if FGF signaling is required for fate specification of the progenitor cells lying at both sides of the OF margin (OFM) (they will be referred to as OFM progenitors hereafter). We compared the expression pat- terns of Pax2, Pax6, Vsx2 (formerly known as Chx10) and Mitf in both the control and Fgfr-DCK OCs during the period of OF closure. Pax 2 and Pax 6 reciprocally repress each other to regionalize eye primordia in the OC [58]. Pax2 is highly expressed in OFM progenitors, whereas Pax6 shows high expression levels in NR and RPE progenitors but low levels in OFM progenitors (Figure $3 \mathrm{~A}$ and $3 \mathrm{C}$ ). Regions of Pax2 expression gradually shrink as OF closure proceeds, while Pax6-expressing regions expand (Figure 3A and 3C) [59]. Similarly, $V_{s} x 2$ and Mitf reciprocally repress each other to establish complementary expression patterns and specify the NR and RPE domains within the OC $[60,61]$ (Figure $3 \mathrm{~B}$ and
E10.5
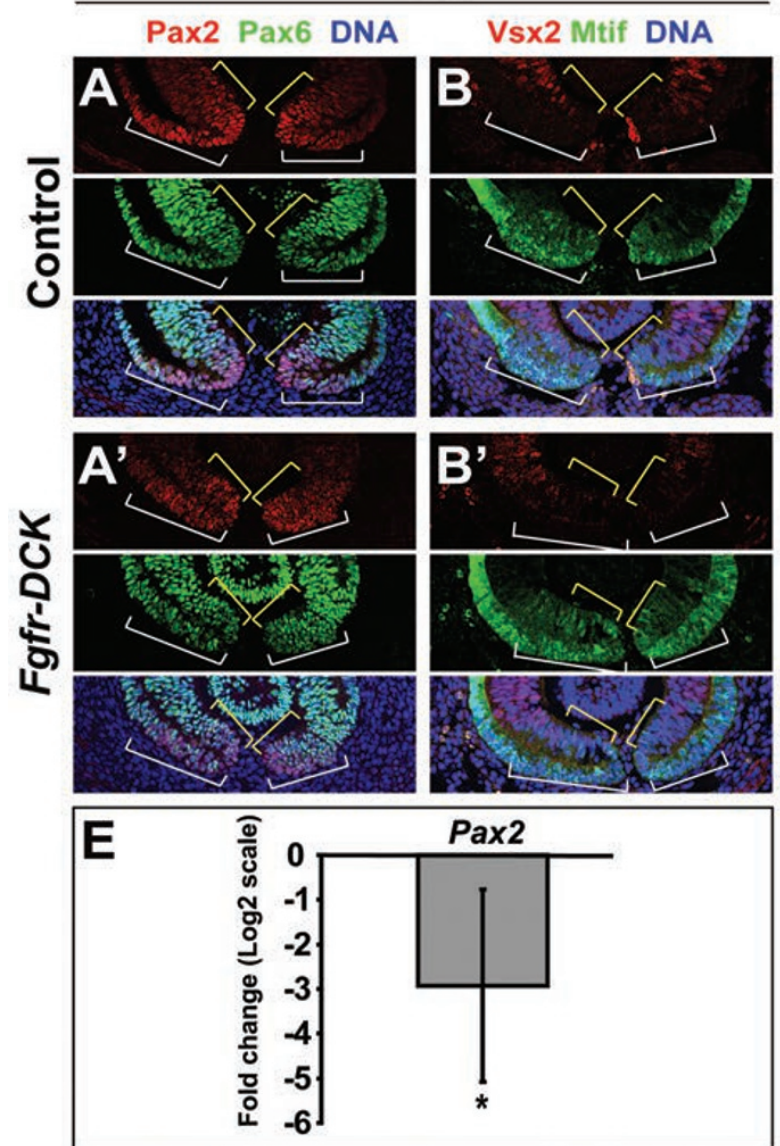

E11.5
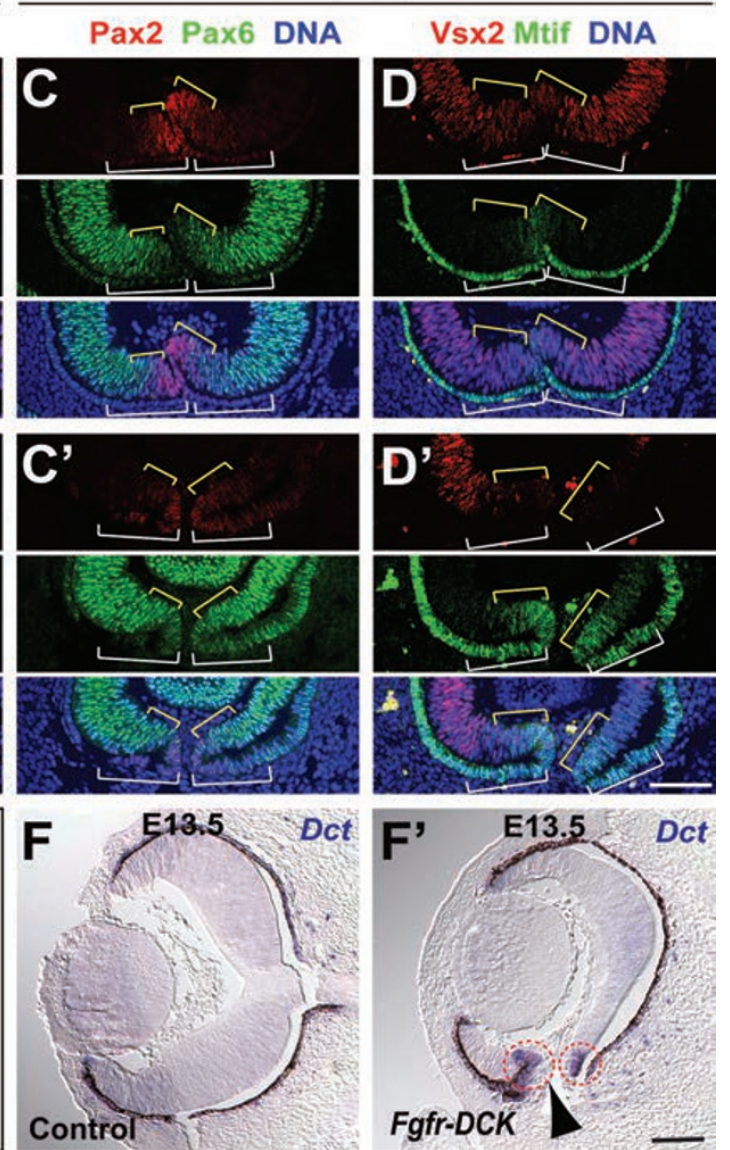

Figure 3 Fgfr-DCK OFM progenitor cells are defective in cell fate switches. (A, A') At E10.5, Pax2 is highly expressed, and Pax6 is moderately repressed in the OFM of both control (A) and Fgfr-DCK mutant (A') OCs. (B, B') At E10.5, Mitf is moderately expressed, and Vsx2 is mostly repressed in the OFM in both control (B) and Fgfr-DCK mutant (B') OCs. (C, C') At E11.5, Pax2 is prematurely downregulated, and Pax6 is abnormally upregulated in the OFM of the Fgfr-DCK mutant OC (C') in comparison with the control OC (C). (D, D') At E11.5, Mitf and Vsx2 are abnormally upregulated and downregulated, respectively, in the inner OFM layer of the Fgfr-DCK OC (D') in comparison with the control OC (D). (E) qPCR results show that Pax2 mRNAs are dramatically downregulated in the Fgfr-DCK mutant OCs in comparison with the control OCs. ${ }^{*}$ denotes $P$ $<0.01$. (F, F') At E13.5, Dct mRNAs are abnormally upregulated in the unclosed OF region of the Fgfr-DCK mutant OCs (red dotted circles). In A-D', white brackets highlight the outer OFM layer, and the yellow brackets highlight the inner OFM layer. Scales bars are $100 \mu \mathrm{M}$. 
3D). At E10.5, the expression patterns of Pax2, Pax6, Vsx2, and Mitf proteins do not show any obvious differences between the control and the Fgfr-DCK mutant OCs, indicating that OFM progenitors still develop normally at E10.5 (Figure 3A-3B'). However, at E11.5, Pax2 expression levels and domains in the $F g f r-D C K$ mutant OFM are dramatically reduced in comparison with those in the control, indicating that FGF signaling is required for maintaining the OFM progenitor fate (Figure 3C and $3 \mathrm{C}^{\prime}$ ). In addition, we used qPCR to further confirm that Pax 2 mRNA levels are indeed dramatically downregulated in the Fgfr-DCK mutant OCs (Figure 3E). Consistent with the mutually repressive relationship between Pax 2 and Pax6, Pax6 expression is increased in the mutant OFM progenitors (Figure 3C'). Similarly, Vsx2 and Mitf expression patterns also changed at the $\mathrm{Fg} f \mathrm{r}$ $D C K$ mutant OFM in comparison with the control OFM (Figure $3 \mathrm{D}$ and $3 \mathrm{D}^{\prime}$ ). Mitf is abnormally upregulated in the inner layer of the mutant OFM (Figure 3D and 3D'), whereas Vsx2 is downregulated in the inner layer of the $F g f r$-DCK OFM (Figure 3D and 3D'). These results have clearly demonstrated that FGF signaling is required for maintaining the OFM progenitor fate and thus controlling the properly timed cell fate switches.

Pax6 and Mitf are important for the establishment and maintenance of the RPE fate $[35,59,60,62]$. The upregulation of Pax6 and Mitf in the inner layer of the Fgfr-DCK mutant OFM progenitors would lead to the RPE fate instead of the NR fate. Dct is expressed in the mature RPE, serving as a mature RPE marker (Figure 3F). Indeed, the E13.5 Fgfr-DCK progenitors at the unclosed OFM, regardless of their location in the inner or outer layer, develop into RPE cells based on Dct expression and pigmentation (Figure $3 \mathrm{~F}^{\prime}$ ). Taken together, our results demonstrate that FGF signaling is required for maintaining Pax2 expression in OFM progenitors and regulating OFM fate switches.

FGF signaling is required for controlled highly coordinated morphological changes of OFM progenitors

In the Fgfr-DCK mutant retina, we consistently observed that NR and RPE layers are frequently separated from each other in the OF region, raising an interesting possibility that those mutant OFM progenitors exhibit adhesion defects, cell morphological defects or both (Figure 1G, 1H and 1M). In wild-type mice, the OF closure was previously reported to occur at around E11, starting in the middle of the proximal-distal (P-D) axis $[4,63]$ (Figure 4A-4C). At E10.5, the fissure between the nasal and temporal retinas is widely open, and is still filled with migrating mesenchymal cells (Figure 4D). At E11.5, two OF edges perfectly align against each other to fuse at the middle of the P-D axis (Figure 4F and 4G), while it remains open at both its most distal and proximal end (Figure 4E and 4H). At different positions along the P-D axis of the fissure, there are dynamic and yet highly coordinated changes in cellular morphologies of OFM progenitors: the OFM progenitor cells on the outer layer at the mid-point of the P-D axis become more cuboidalshaped than those at either the distal or proximal position (Figure 4E-4H). Interestingly, when the two sides of the fissure align against each other, the temporal side always stacks on the top of the nasal side (Figure $4 \mathrm{~F}$ and $4 \mathrm{G}$ ). Such nasal-temporal asymmetry can be used to reliably distinguish the nasal and temporal sides, but it remains unclear whether it is important for the OF closure. At E11.5, the fusion begins around the 'folding point', where the inner and the outer layers meet, from the inner layer to the outer layer [4, 63] (Figure 4G and 4J). This can be easily monitored by dissolution of the lamininpositive basal membrane on the OFMs (Figure $4 \mathrm{~J}$ ). At E12, the OF fusion is complete: the OFM progenitors on the inner layer are integrated into a continuous NR layer, while those on the outer layer form a continuous RPE layer (Figure 4I). Therefore, the fissure closure is accompanied by highly coordinated cell morphological changes of OFM progenitors.

Compared with the control OFM progenitors, the E11.5-E12 Fgfr-DCK mutant OFM progenitors exhibited five major differences. First, the two mutant fissure margins at the mid-point of the P-D axis fail to touch each other, and their basal membrane remain intact, which is in stark contrast with the control in which two OFMs align against each other perfectly, while the basal membrane begins to dissolve (Figure $4 \mathrm{~J}$ and $4 \mathrm{~K}$ ). Second, the open mutant OF still allows mesenchymal cells to migrate into the $\mathrm{OC}$ in contrast with the closed control fissure preventing the entry of mesenchymal cells into the OC (Figure 4L and 4M; The mesenchymal cells are labeled by Pitx 2 expression). Third, the mutant OFM progenitors on both the inner and outer layers show morphological defects in comparison with those control counterparts. The mutant OFM progenitors on the inner layer are shorter in length than those in the control, while the OFM progenitors on the outer layer are taller than those control counterparts (Figure 4N and 4O). Fourth, the temporal side of the OFM are positioned downward under the nasal side, which is exactly opposite to that in the control (Figure 4K, 4M, 4O and 4Q). Finally, the inner and outer layers are more frequently separated from each other in the mutant OFM region than those in the control (Figure 4O and 4Q).

The contractile forces generated by acto-myosin activity have been shown to regulate actin cytoskeletons, cell- 

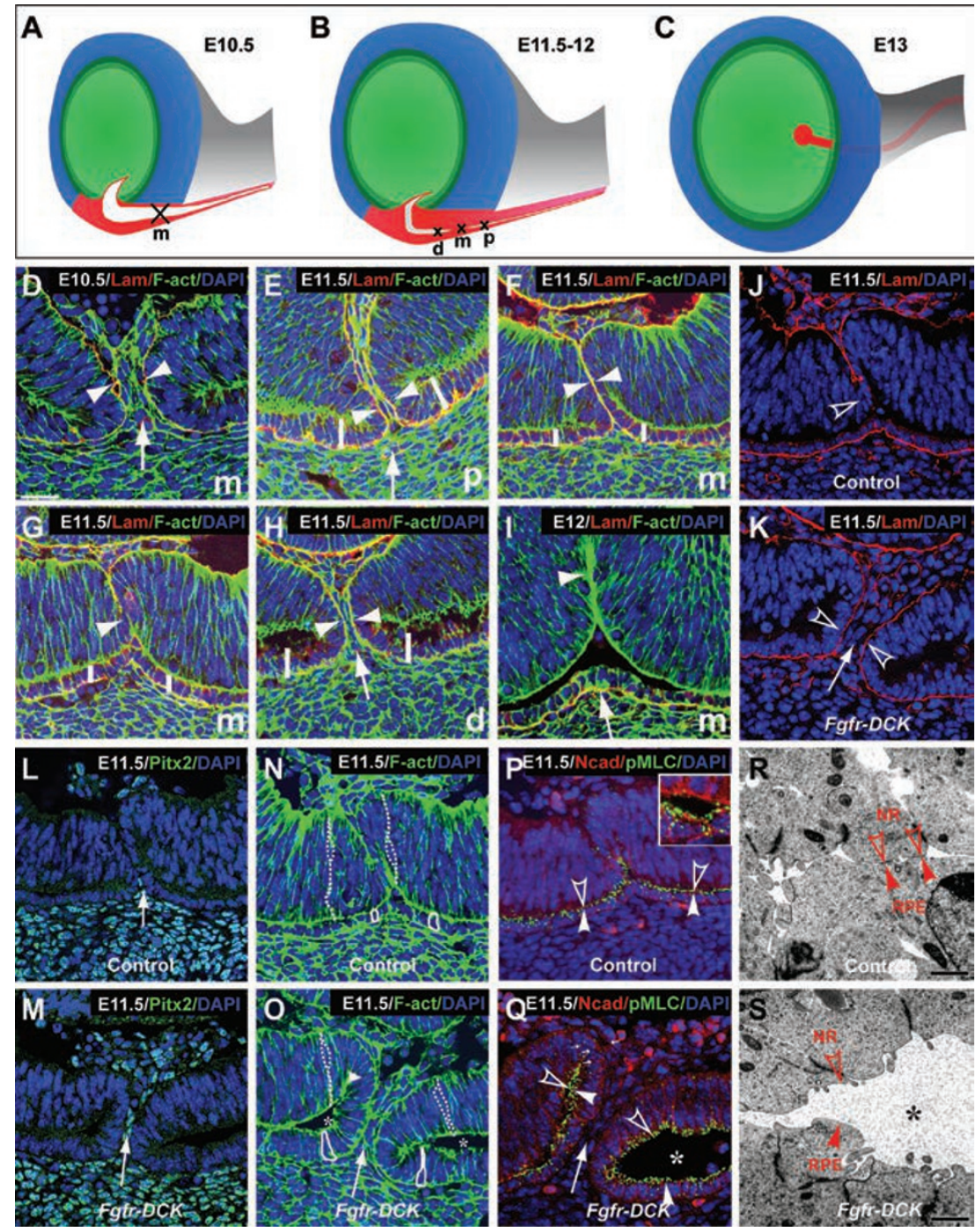

Figure 4 The OFM progenitors of Fgfr-DCK mutant OCs exhibit morphological defects. (A-C) Schematic presentation of developing OCs around the OF closing period (blue: RPE; green: the NR; red in A and B: the OFM; red in C; the optic nerve). The sampling positions along the P-D axis of the OF used for D-I are indicated by $x$ (d: distal; m: middle; p: proximal). (D-I) Wild-type OCs at different developmental stages, which are labeled for Laminin (red, basal membrane) and for filamentous actin (F-act, green), show that the edges of the middle portion of the E11.5 OF has aligned perfectly against each other (F) or has begun the fusion process by dissolving the basal membrane (arrowhead, G), while the same OFs at the proximal (E) and distal $\mathbf{( H )}$ regions still leave an opening by the arrow. Two fissure edges in $\mathbf{E}, \mathbf{F}$ and $\mathbf{H}$ are denoted by arrowheads, whereas the open fissure is indicated by an arrow throughout this figure. White bars in $\mathbf{E}$ to $\mathbf{H}$ highlight the height of the outer RPE layer. At E12 (I), the fissure finishes closing by forming continuous layers of RPE (arrow) and NR (arrowhead). (J, K) E11.5 control and Fgfr-DCK OCs labeled for Laminin show that the control fissure has begun the fusing process (loss of the basal membrane indicated by open arrowhead in $\mathbf{J}$ ), while the edges (open arrowheads in $\mathbf{K}$ ) of the Fgfr-DCK mutant OF still leave a gap indicated by an arrow. (L, M) In the E11.5 control OC, Pitx $2^{+}$mesenchymal cells have stopped migrating into the vitreous space due to the closing of the OF (arrow), while those in the E11.5 Fgfr-DCK mutant OCs still migrate into the vitreous space through the open fissure (arrow). (N, O) E11.5 control and Fgfr-DCK OCs, which are labeled for F-actin and DNA, show that the mutant OFM progenitors in inner (dashed lines) and outer layers (solid lines) are shorter and taller than those corresponding control ones, respectively. ${ }^{*}$ in $\mathbf{O}, \mathbf{Q}$ and $\mathbf{S}$ mark the gap left by the two separated layers. (P, $\left.\mathbf{Q}\right) \mathbf{E} 11.5$ control and Fgfr-DCK mutant OCs, which are labeled for N-cadherin (red) and pMLC (green), show that pMLC accumulate on the apical side of Fgfr-DCK mutant OFM progenitors on both inner (open arrowhead) and outer (closed arrowhead) layers, which is in contrast with the control OFM progenitors, which express pMLC only on the outer layer. The insert in $\mathbf{P}$ highlights the region where inner and outer layers are separated from each other due to histological artifact. (R, S) EM graphs show that some junctions in the control OF hold inner and outer layers together, while the two layers are separated from each other in the Fgfr-DCK mutant OC. The scale bar in $\mathbf{D}$ is $25 \mu \mathrm{M}$, whereas those in $\mathbf{R}$ and $\mathbf{S}$ are $1 \mu \mathrm{M}$. 

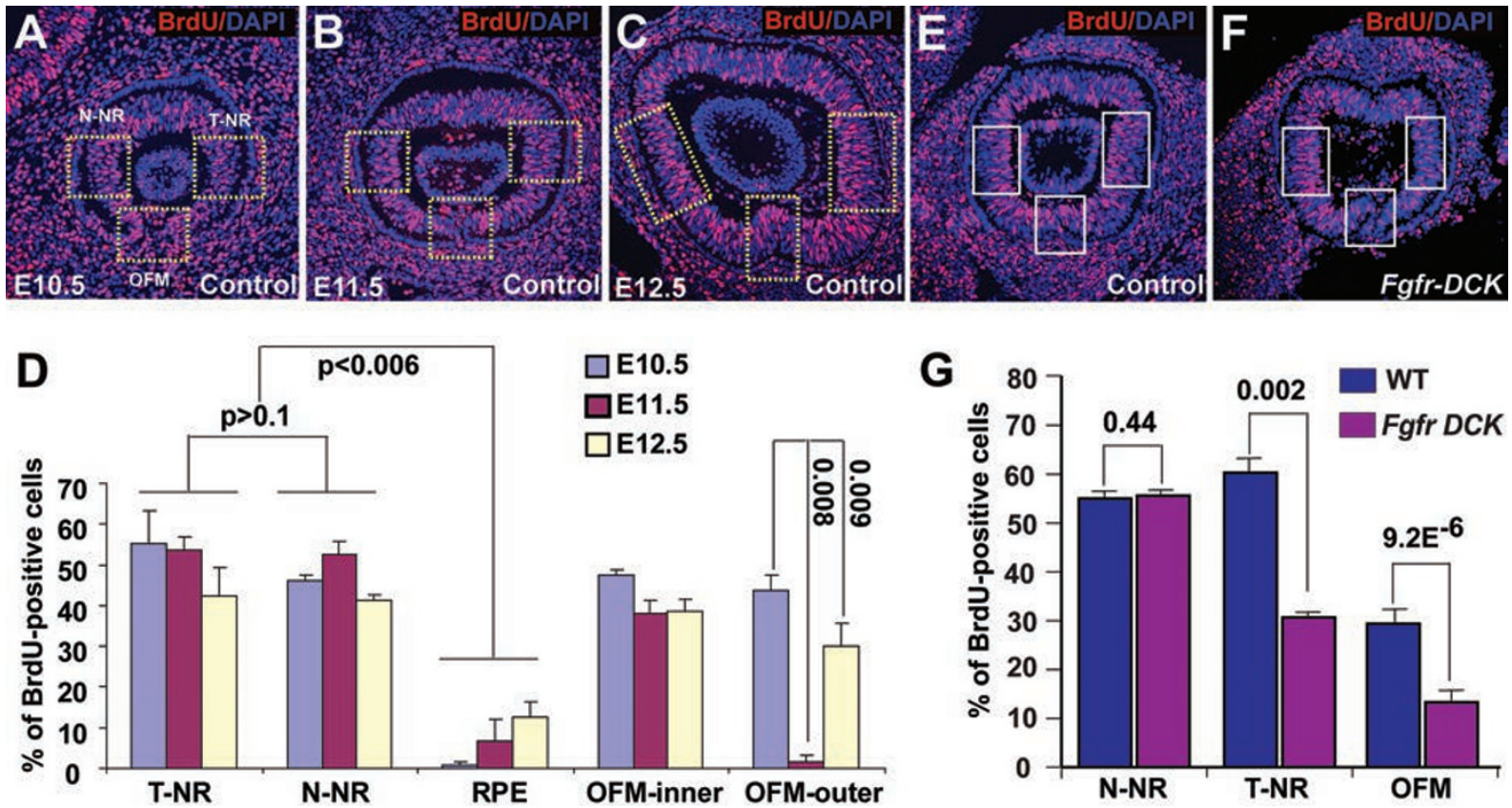

Figure 5 FGF signaling controls the proliferation of OFM and NR progenitors. (A-D) Two-hour BrdU labeling of wild-type E10.5, E11.5 and E12.5 OCs, which are stained for BrdU and DNA. The percentages of BrdU-positive cells in highlighted areas (dashed lines) of the nasal neural retina (N-NR), temporal neural retina (T-NR), RPE, inner layer at the OFM and outer layer at the OFM are quantified, and the quantification results are shown in D. (E-G) E11.5 control and Fgfr-DCK mutant OCs, which are labeled by two-hour BrdU incorporation. The quantitative results on the BrdU-positive cells in the highlighted areas (boxed) are shown in $\mathbf{G}$.

cell adhesion and cell morphological changes, and are essential for epithelial morphogenesis in multiple organisms $[64,65]$. To explore the possibility that acto-myosin is involved in the regulation of the OF morphogenesis, we examined the expression of phosphorylated myosin light chain (pMLC) in the control and mutant Fgfr$D C K$ OFM progenitors. pMLC is an indicator for local acto-myosin activation [64]. In the control eyes, pMLC accumulation is enriched in the OFM progenitors on the apical and lateral sides of the outer layer but not the inner layer (Figure 4P). This is consistent with recently published results from in vitro cultured OCs [66]. In contrast, pMLC accumulates in the $F g f r-D C K$ mutant OFM progenitors on the inner layer in addition to those on the outer layer, and the accumulation is concentrated on apical and lateral sides of both the layers (Figure 4Q). Like in the rest of the control OC, N-cadherin is expressed in OFM progenitors on the apical and lateral sides of inner and outer layers, which might contribute to adhesion between inner and outer layers as well as within the same layers in the OFM region (Figure 4P). Consistently, adherens junction (AJ)-like structures can be detected between inner and outer layers as well as between the progenitors of the same layers in the OFM region (Fig- ure $4 \mathrm{R}$ ). In contrast, the AJ-like junctions between NR and RPE layers are often lost in the $F g f r-D C K$ mutant OFM progenitors (Figure 4S). Therefore, the abnormal distribution of acto-myosin activity might contribute to the cell morphological and adhesion defects of the Fgfr$D C K$ mutant OFM progenitors and consequently the failure of OF closure.

\section{FGF signaling controls the proliferation of OFM and NR progenitors}

Cell proliferation has been suggested to play a critical role in driving tissue morphogenesis $[67,68]$. To this end, we examined cell proliferation of OFM progenitors, NR progenitors and RPE progenitors during the period of the OF closure. During the E10.5-E12.5 period, about $50 \%$ of NR progenitor cells on either the nasal side or the temporal side of the wild-type retina are positive for BrdU labeling following two hours of BrdU incorporation, indicating that NR progenitors on both the nasal and temporal sides proliferate at similar and rapid rates (Figure 5A-5D). In contrast, RPE progenitors outside the OF region increase their BrdU-labeling rates from $0.8 \%$ to $12.5 \%$ during E10.5-E12.5, indicating that RPE progenitors proliferate dynamically but at much lower rates 
than NR progenitors (Figure 5A-5D). For OFM progenitors, about $40 \%$ of those on the inner layer are positive for BrdU from E10.5 to E12.5, indicating that OFM progenitors on the inner layer proliferate fast like NR progenitors during the OF closure (Figure 5A-5D). Surprisingly, the OFM progenitors on the outer layer show dynamic proliferation patterns during the same period: $43.6 \%, 1.7 \%$ and $30.0 \%$ of the OFM progenitors on the outer layer are positive for BrdU labeling at E10.5, E11.5 and E12.5, respectively (Figure 5D). Interestingly, the time when those OFM progenitors on the outer layer stop proliferation is also the moment when the fissure closure actually takes place, and in contrast they proliferate more rapidly before and after the OF closure (Figure 5D). These observations prompted us to consider the possibility that dynamic proliferation patterns of OFM progenitors might contribute to the OF closure.

One of the most obvious phenotypes is that the Fgfr$D C K$ mutant OCs and eyes are smaller than controls (Figure 1A and 1B). In addition, in the Fgfr-DCK OCs, the retina on the nasal side is thicker than that on the temporal side, which is closely correlated with the fact that $F g f r$ - $D C K$ mutant retinal progenitors are absent from the nasal side (Figure 1G, $1 \mathrm{~J}$ and $1 \mathrm{~K}$ ). Furthermore, there is no increase in apoptotic retinal progenitor cells in the Fgfr-DCK mutant OCs in comparison with the control OCs based on TUNEL labeling (Supplementary information, Figure S5). These observations support the possibility that FGF signaling regulates retinal progenitor cell proliferation. As an internal control, NR progenitors on the nasal side of the control and Fgfr-DCK mutant OCs at E11.5 have the same percentages of BrdU-positive cells, indicating that BrdU labeling efficiencies between the control and mutant OCs are similar (Figure 5E-5G). In contrast, NR progenitors on the temporal side of the $F g f r-D C K$ mutant OCs have much lower percentages of BrdU-positive cells than those in the control (Figure 5E$5 \mathrm{G})$. Consequently, in the Fgfr-DCK mutant OCs, wildtype NR progenitors on the nasal sides and those mutant progenitors on the temporal sides proliferate at significantly different rates, which might also contribute to the misalignment of two OFMs. Similarly, the OFM progenitors in the $\mathrm{Fg} f \mathrm{r}$-DCK mutant OCs proliferate much slower than those in the control OCs (Figure 5E-5G). These results demonstrate that FGF signaling regulates NR and OFM progenitor cell proliferation.

FGF signaling controls expression of the genes in OFM progenitors that are important for cell proliferation, fate determination and actin dynamics

To better understand how FGF signaling controls the OF closure at the molecular level, we used microarrays to compare the gene expression profiles between normal and Fgfr mutant E11.5 OFM progenitors. The OFM progenitors from the Fgfr-DCK mutant and control E11.5 OCs were isolated using a laser-assisted micro-dissection microscope (a total of 12 control and 12 mutant OCs were used) to prepare RNAs for microarray analysis (Figure 6A and 6B). Based on a twofold difference, 325 genes are upregulated and 476 genes are downregulated in $F g f r$ mutant OFM progenitors in comparison with the controls. Some of the gene expression changes between control and mutant OFM progenitors were confirmed by quantitative PCRs, of which two are shown in Figure 6D. Many of the genes regulated by FGF signaling are functionally related to cell cycle progression, retinal cell fate determination, extracellular matrix (ECM)-mediated adhesion, and cytoskeleton regulation, which could potentially offer insight into the molecular mechanisms of OF closure regulated by FGF signaling (Figure 6C and Supplementary information, Table S1):

Cell cycle regulators In the Fgfr-DCK mutant OFM progenitors, the expression of Cyclin D1 (Ccnd1) and D2 (Ccnd2), Cdc25a, Cdc2a (Cdk1), c-Myb, inner centromere protein (Incenp) and abnormal spindle-like microcephaly associated (Aspm) mRNAs decrease by more than twofold in comparison with the controls (Figure 6C and Supplementary information, Table S1).

Cytoskeleton regulators Eight known actin regulators, including RhoA, Enabled homolog (Enah), Coronin1C (Coro1C), Palladin (Palld), and Profilin1 (Pfn1), are downregulated by more than twofold in the $F g f r$ mutant OF progenitors (Figure 6C and Supplementary information, Table S1). These downregulated actin regulators are known to regulate actin dynamics, cytoskeleton remodeling, adhesion, and thus cell motility [69-73]. Interestingly, no obvious changes for tubulin genes and microtubule regulators, which are also known to regulate cell motility, have been observed in the mutant OF progenitors.

OFM cell fate determinants In addition to Pax2 downregulation (Figure 3), our microarray results show that Vsx2 and Vax1 mRNAs are also downregulated more than twofold in Fgfr mutant OFM progenitors in comparison with the control progenitors (Figure 6C and Supplementary information, Table S1). Vax1 is a homeoboxcontaining protein that is specifically expressed in OFM progenitors, and a mutation in Vaxl causes coloboma formation in mice $[8,9]$. $V_{s x} 2$ and Mitf mutually repress each other's expression in the developing OC, while Pax6 and Pax2 also mutually repress each other's expression. As expected, Mitf and Pax6 mRNAs are up- 


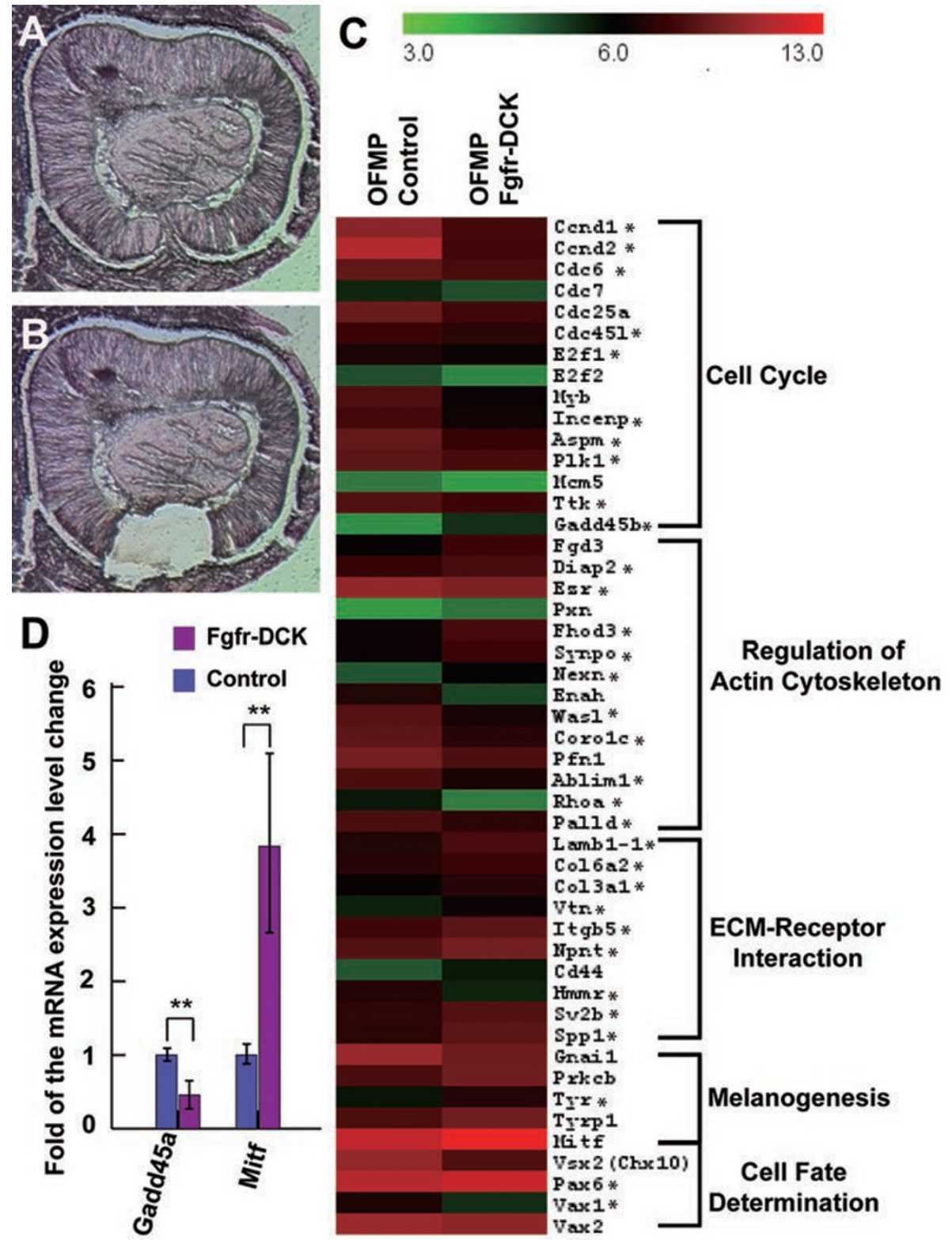

Figure 6 FGF signaling controls the expression of the genes in the OFM important for cell proliferation, cytoskeleton regulation, and cell fate determination. (A, B) Images of the E11.5 control OC before (A) and after (B) the OFM progenitors are removed by microdissection. The OFM progenitors of E11.5 control and Fgfr-DCK OCs are analyzed for gene expression profiles using Affymetrix microarray. (C) Heatmap representation of important regulators for cell cycle, actin cytoskeleton, ECMreceptor interaction, melanogenesis and cell fate determinants, which are differentially expressed in the control and Fgfr-DCK OFM progenitors. (D) qPCR confirmation of two differentially expressed genes.

regulated in Fgfr-DCK mutant OFM progenitors in comparison with the corresponding control ones (Figure 6C and Supplementary information, Table S1). In addition, microarray results have further confirmed our mRNA in situ results that mRNAs for Vax2, a ventral retinal cell marker, show no change (Figure 6C), further supporting the idea that FGF signaling maintains OF progenitor cell fate but not the general ventral cell fate.
To further explore if any of these genes are potentially direct target genes of FGF signaling, we used a bioinformatics approach to find the putative Pea3/Erm binding site(s) (core consensus AGGA(A/T)) in the promoter of the genes that are regulated by FGF signaling in OFM progenitors. As shown earlier, FGF signaling is required for maintaining the expression of $\mathrm{pERK}$, the activated form of MAPK, which phosphorylates Ets domain- 
containing proteins and thereby represses or activates transcription of their target genes. Two Ets-containing proteins, Pea3 and Erm, can function downstream of FGF receptors to control gene expression [74]. Among the FGF-regulated genes in Figure 6C, the majority of them have at least one Pea3/Erm binding site, suggesting that they are potentially direct FGF targets (Figure 6C and Supplementary information, Table S1). In the future, it will be important to investigate which genes are true direct transcriptional targets of FGF signaling in OFM progenitors.

FGF signaling is required for the maintenance of the OS

As mentioned earlier, the Fgfr-DCK mutant eyes lack the optic nerve. To examine the development of the optic nerve, we checked the expression patterns of Pax2, the marker for OS progenitor cells, in the control and $\mathrm{Fg} f$ r$D C K$ mice. At E11.5, Pax2 expression in the ventral retina of the Fgfr-DCK mutant eye is reduced in comparison with that in the control eye, however, its expression in the OS remains normal in the mutant eye (Supplementary information, Figure S6A and S6B). At E13.5, Pax2 remains expressed in the optic disc of the control eye, but the Pax2-positive cells are completely absent in the Fgfr$D C K$ mutant OC (Supplementary information, Figure $\mathrm{S} 6 \mathrm{C}$ and S6D). In addition, although Pax2-positive OS cells can still be found in the E13.5 Fgfr-DCK mutant eye, they are dramatically reduced in comparison with the control eye (Supplementary information, Figure S6C and S6D). At E15.5, the Pax2-positive optic disc and optic nerve astrocytes are readily detected in the control eye, but are completely absent in the mutant eye (Supplementary information, Figure S6E and S6F). These results indicate that FGF signaling is required for maintaining the optic stalk.

\section{FGF signaling regulates the development of the RGCs}

Studies in zebrafish and chicken have indicated the role of FGF signaling in initiating RGC development in addition to its role in promoting NR fate $[31,75]$. In Fgfr-DCK mice, the abnormal RPE fate switch is only restricted to the margin of the unclosed OF, while Fgfr$D C K$ mutant inner retinal progenitor cells outside the OFM still adopt the NR progenitor fate, based on the absence of pigmentation and cell morphology (Figure $3 \mathrm{~F}^{\prime}$ ), which gives us an opportunity to test whether initiation of RGC development is affected by defective FGF signaling in mice. We examined the mRNA expression patterns of Math 5 and $B r n 3 b$, two transcription factors determining the retinal precursor RGC competent state, and RGC terminal differentiation, respectively $[21,76]$. At E13.5, Math5 and Brn3b are expressed throughout the NR in the control OCs (Figure 7A and 7C). However, the expression levels of both Math 5 and Brn $3 b$ are either absent or dramatically reduced on the temporal side of the Fgfr-DCK OCs, while the expression levels on nasal OCs remain relatively normal (Figure 7B and 7D). Since the defects in the expression of Math5 and Brn $3 b$ are well correlated with the area where FGF receptors are most efficiently deleted (Figure $1 \mathrm{~J}$ ), these results indicate that FGF signaling is required for the initiation of RGC development.

Due to the highly mosaic nature of Cre-mediated deletion, RGCs are still generated in Fgfr-DCK OCs based on abundant Brn $3 b$ expression on the nasal part of the OCs (Figure 7D). As we described above, the optic nerve does not form in $F g f r-D C K$ mice due the degeneration of the OS. To examine the fate of the axons of RGCs in the absence of the OS in Fgfr-DCK eyes, we immunostained the E15.5 control and $F g f r-D C K$ eye sections with an antibody recognizing Tuj1. Our results show that RGC axons in the control eye form the optic nerve exiting the optic disc, but the axons of the remaining RGCs in the mutant eye exit the unclosed fissure and are mis-targeted to the subretinal space (Figure 7E and 7F). To trace the fate of the unclosed OF and retinal neurons in $F g f r-D C K$ eyes, we collected one-month-old and seven-month-old eyeballs. The H\&E-stained eye sections show that the fissure remains unclosed in the one-month-old Fgfr-DCK mutant eye (Supplementary information, Figure S7A), and the retina gradually degenerates with time, which is evident in the seven-month-old mutant eye (Supplementary information, Figure S7B). These results suggest that FGF signaling directly or indirectly regulates the maintenance of retinal neurons.

\section{Discussion}

Studies of lower vertebrates and cultured organs have linked FGF signaling with a variety of processes during retinal development ranging from initial eye field formation to retinal neuron differentiation. However direct genetic evidence of how FGF signaling regulates mammalian retina development is still lacking. In this study, we conditionally deleted Fgfrl and Fgfr2 from the developing OC to show that FGF signaling controls the OF closure process and the initiation of RGC development. Although a number of genes have been implicated in coloboma formation, it remains largely unclear how the OF closure fails at the cellular level. In this study, we show that FGF signaling controls the OF closure possibly by regulating the proliferation, morphological changes and cell fate switches of OFM progenitors. The OF closing process is accompanied by highly coordi- 

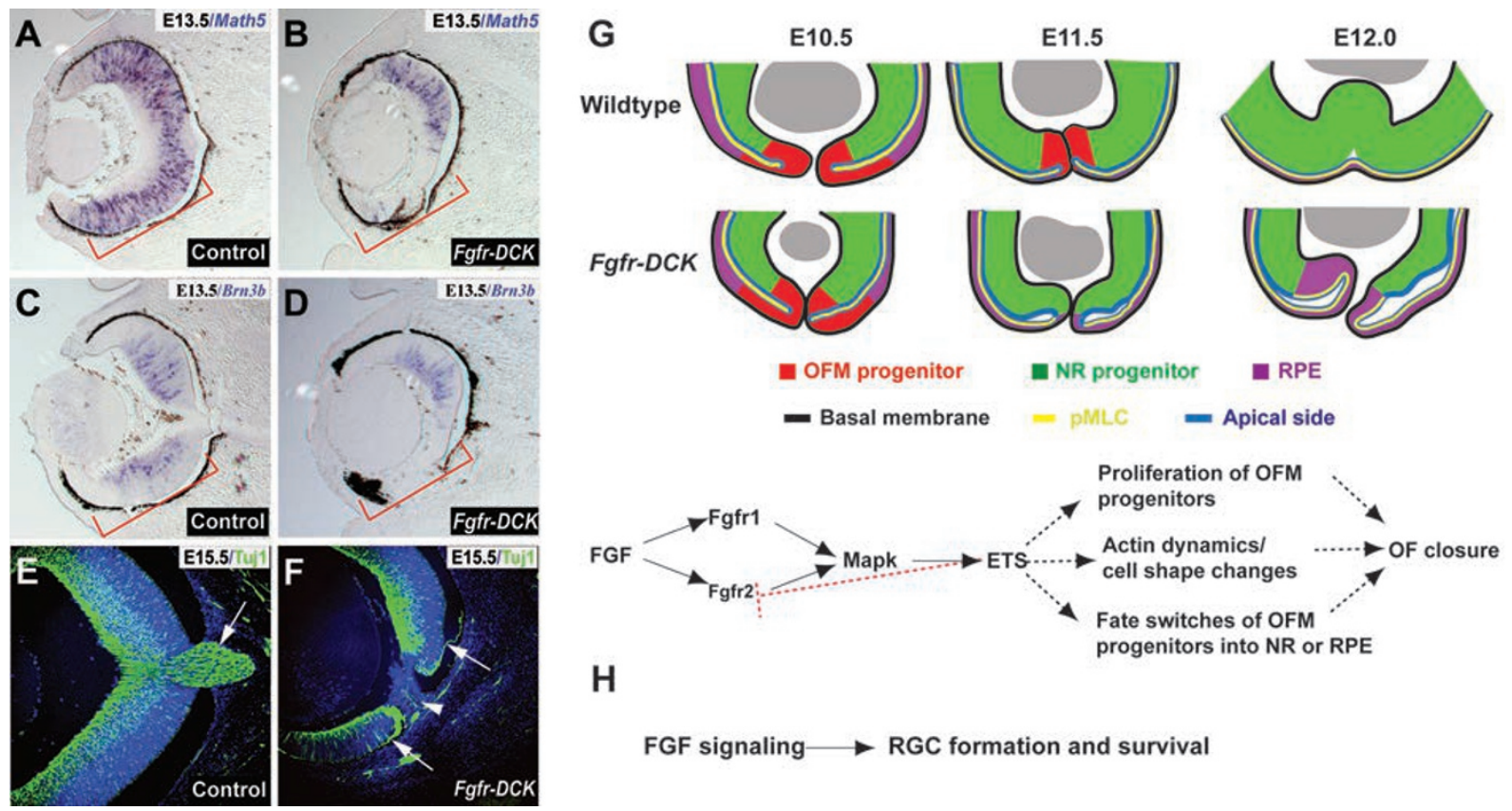

H

FGF signaling $\longrightarrow$ RGC formation and survival

Figure 7 FGF signaling regulates the development of RGCs, and the model for the function of FGF signaling during retina development. (A, B) Math5 mRNA in situ results show that Math5-positive RGCs decrease more dramatically in the temporal side around the unclosed fissure area (red bracket) in the Fgfr-DCK eye than in the area distant from the unclosed fissure. (C, D) Brn3b in situ results show that Brn3b-positive RGCs decrease more dramatically in the temporal side around the unclosed fissure area (red bracket) in the Fgfr-DCK eye than in the area distant from the unclosed fissure. (E, F) The Tuj1-positive RGC axons project to the brain through the optic nerve (arrow) in the control eye (E), while they are mis-routed to the subretinal space (arrows) via the unclosed fissure (arrowhead) in the Fgfr-DCK eye (F). (G) The model explaining how FGF signaling control the OF closure. (H) FGF signaling regulates the development of RGCs.

nated cell proliferation, morphological changes and cell fate switches in OFM progenitors. In the absence of FGF signaling, OFM progenitors fail to undergo coordinated cell proliferation, morphological changes and cell fate switches. Furthermore, FGF signaling is required for maintaining the expression of the genes that are important for cell cycle progression, cytoskeleton dynamics and retinal progenitor fate specification. Based on our detailed analyses of cellular events and gene expression in both wild-type and Fgfr mutant eyes, we propose a working model that the coordinated cell proliferation, cell morphological changes and cell fate switches may collectively contribute to the OF closure, and that defective FGF signaling in the retina leads to coloboma formation by disrupting such highly orchestrated cellular events (Figure 7G). Therefore, this study has provided important insight into how FGF signaling coordinates independent cellular events to control the OF closure.

The OF closure is accompanied by a series of highly coordinated cellular events

The OF closure process begins at the mid-point of the
OF and proceeds rapidly in both directions toward the most distal and proximal points $[4,63]$ (Figure 4). In this study, we have revealed three important cellular events associated with the OF closing process (Figure 7). First, OFM progenitors are switched to NR and RPE progenitors in a location- and time-dependent manner during the OF fusion. Prior to the OF fusion, OFM progenitors express Pax2, while NR and RPE progenitors outside the OF area express Vsx2 and Mitf, respectively [4] (Figure 3). Pax6 is expressed in all the NR and RPE progenitors outside the OF, and is repressed in OFM progenitors [77] (Figure 3). During the fusion process, OFM progenitors on both inner and outer layers gradually retreat Pax2 expression to the edge of the OF. Likely due to loss of Pax2 repression, the OFM progenitors on the outer layer gain Pax6 and Mitf expression and develop into RPE progenitors, while those on the inner layer start Vsx2 and Pax6 expression and develop into NR progenitors. Genetic studies have shown that $\operatorname{Pax} 2$ and $\operatorname{Pax} 6$ mutations cause coloboma in both humans and mice $[5,7,59,78]$. Therefore, it is reasonable to assume that proper cell fate switches are one of the requirements for OFM progeni- 
tors to integrate into the NR and RPE layers following the fusion.

Second, OFM progenitor cells on inner and outer layers undergo distinct but apparently coordinated cell morphological changes just before the OF closure. The closer the OFM progenitors are to the fusion point of the OF, the shorter those on the outer layer become, and the longer those on the inner layer become. Such highly coordinated morphological changes in both layers could conceivably help to correctly position the two OFMs to facilitate the fusion, and could also ensure that the newly fate-switched OFM progenitors have comparable cellular morphologies to their neighboring NR or RPE progenitors. There are strong AJs between the OFM progenitors on the same layer and weak AJs between inner and outer layers (Figure 4R). Weak AJs between inner and outer layers in the OF region can keep the two layers together and meanwhile also allow them to move against each other, while strong AJs between OFM progenitors on the same layers can maintain layer integrity by preventing ruptures. These AJ junctions are most likely formed by $\mathrm{N}$-cadherin in the developing OFM progenitors. N-cadherin-mediated cell adhesion in zebrafish has also been shown to be important for the OF closure [79]. Therefore, adhesion and dynamic cytoskeleton changes may contribute to cell morphological changes and thus the OF closure.

Third, balanced and rapid proliferative activity of the NR progenitors on both temporal and nasal sides of the OC may help align the two OF lips against each other, facilitating the fissure fusion. NR progenitors on the nasal and temporal side proliferate at similar rates from E10.5 to E11.5. Interestingly, OFM progenitors on the outer layer are highly proliferative at E10.5 and cease proliferation at E11.5, which is just before the closing process begins, while those progenitors on the inner layer continue to proliferate at high rates. The biological significance of the dynamic proliferation rates for OFM progenitors on the outer layer remains unclear. Proper proliferation of NR and OFM progenitors is likely important for the OF closure because a mutation in Phactr4, which encodes a phosphatase that inhibits cell-cycle progression, drives excessive proliferation of NR progenitors, leading to coloboma formation [17].

Our study has revealed the cellular events that accompany the OF closure. The changes in these cellular events during the closing process implicate them in driving, maintaining or supporting the OF closure. Although the specific roles of these cellular events in the OF closure remain to be investigated by directly disrupting each of them at a time, our genetic study on FGF signaling in the developing $\mathrm{OC}$ has revealed their potentially important roles in the regulation of the $\mathrm{OF}$ closure.

FGF signaling controls the OF closure possibly by orchestrating multiple cellular events via pERK signaling

Although multiple signaling pathways, such as Shh, BMP and JNK, have been shown to be involved in the regulation of the OF closure, they appear to regulate the expression of cell fate determinants including Pax2, Vax 1 and $\operatorname{Vax} 2$ in OFM progenitors $[3,12,80]$. Mutations in Pax2, Vax1 and Vax2 cause the failure in the OF closure and coloboma formation $[5,7,8,54,78]$. However, it remains unclear how they control the cellular events associated with the OF closure. In this study, we have revealed that FGF signaling regulates cell proliferation and morphological changes of OFM progenitors during the OF closure in addition to Pax2 and Vax1 expression.

FGF signaling regulates the maintenance of the OFM fate and proper switches of OFM progenitors into RPE and NR progenitors by controlling the expression of OFM fate determinants Pax2 and Vax1. Although OFM progenitors are properly established in the $F g f r$ mutant OCs at E10.5 based on Pax2 expression, they lose their cell identity prematurely in the E11.5 mutant OCs based on Pax 2 and Vax1 expression, indicating that FGF signaling is required for maintaining the OFM progenitor fate. Early studies have shown that Vax 1 and Pax2 can antagonize the expression of Pax6 in OFM progenitors [8]. Consistently, our study has shown that Pax6 expression is upregulated in Fgfr mutant OFM progenitors. FGF signaling is known to be required for maintaining $V s \times 2$ expression and thus repressing Mitf expression in the development of the OC $[35,60,61]$. Consistently, $V_{s x} 2$ and Mitf are downregulated and upregulated in the $F g f_{r}$ mutant OFM progenitors, respectively. As a result, the Fgfr mutant OFM progenitors express Pax6 and Mitf and develop into RPE cells. Therefore, we propose that FGF signaling maintains the OFM progenitor cell fate and controls their fate switches into RPE and NR progenitors by primarily regulating two antagonizing gene pairs Pax2Pax6 and Vsx2-Mtif (Figure 7G).

FGF signaling regulates morphological changes of OFM progenitors possibly by controlling expression of important cytoskeleton regulators. Our microarray results show that the actin cytoskeleton regulators Enah, Pfn 1 and RhoA, which are known to be important for morphological changes and adhesion [69, 81-84], are downregulated in the Fgfr mutant OFM progenitors. In addition, no microtubule regulators are affected in the mutant OFM progenitors. FGF signaling is required for mesodermal invagination and cell morphological changes by regulating actin organization during Drosophila gastrulation [85-90]. Based on these findings, we propose that FGF signaling 
regulates actin cytoskeleton dynamics and thus affects cell morphological changes of OFM progenitors (Figure $7 \mathrm{G})$.

FGF signaling regulates the proliferation of NR and OF progenitors by controlling expression of cell cycle regulators. The Fgfr mutant OF and NR progenitors proliferate much slower than control counterparts based on BrdU labeling, resulting in thinner retina and smaller eye size. In addition, the uneven proliferation on the nasal and temporal sides of the Fgfr mutant retina likely prevents the correct alignment of the two OFMs, contributing to coloboma formation. In this study, we also show that some important cell cycle regulators controlling the G1-S transition, such as Cyclin D1, Cyclin D2 and $c-M y b$, and G2-M transition, such as $C d c 25 a$ and $C d k 1$, are downregulated in the Fgfr mutant OF progenitors. Therefore, our study has shown that FGF signaling controls NR and OFM progenitor proliferation possibly by directly regulating expression of the genes important for both G1-S and G2-M transitions (Figure 7G).

Our study has also suggested that FGF signaling controls the OF closure via regulation of the pERK-Ets pathway in OFM progenitors. The activation of MAPK but not Akt is affected in the $F g f r$ mutant OFM progenitors (Figure 1L-1 $\mathrm{M}^{\prime}$ ), suggesting that FGF signaling activates pERK specifically in OFM progenitors. Although Fgfr 1 and $F g f r 2$ are effectively inactivated on both the temporal and dorsal sides, $\mathrm{pERK}$ remains normal, suggesting that FGF signaling has region-specific functions. Such regionspecific functions may be caused by region-specific expression of FGF ligands or region-specific functional redundancies with other signaling pathways such as EGF signaling. FGF signaling controls the expression of pERK (phosphorylated MAPK), which in turn phosphorylates Ets-containing proteins, Erm and Pea3, to activate target gene expression [91]. Interestingly, some of the downregulated genes in the Fgfr mutant OFM progenitors also contain Erm/Pea3 binding sites (Supplementary information, Table S1). In addition, conditional knockout of Shp 2 in retinal progenitors leads to the defective expression of pERK in retinal progenitors $[32,33]$. Because FGF signaling is required for pERK expression only in OFM progenitors, we speculate that Shp2 may be involved in other signaling pathways, perhaps along with FGF signaling, to maintain $\mathrm{pERK}$ expression in the retina progenitors outside the OF. In summary, our findings suggest that FGF signaling controls various downstream signaling branches in different regions of the retina to regulate cell behavior.

FGF signaling controls the maintenance of the OS and initiation of the $R G C$ development

Retinal coloboma is often associated with defects in the optic nerve, which are often manifested as the extension of the NR into the optic stalk [1]. Our study has offered an alternative way for the eye with coloboma to lose the optic nerve, due to premature loss of the optic stalk. However, the loss of the optic stalk in the Fgfr$D C K$ mutant eye cannot be caused by the loss of Pax 2 expression because Pax 2 mutant mice still develop the optic nerve [7]. Furthermore, the optic stalk has formed initially in the $F g f r$-DCK mutant eye, but degenerates later. These findings suggest that FGF signaling maintains the optic stalk in a Pax2-independent manner.

This study has also revealed an interesting role of FGF signaling in promoting retinal neurogenesis in mice. FGF signaling has long been known to promote NR fate versus RPE fate [28, 92], which is also reflected in $F g f r$ $D C K$ eyes by the ectopically generated RPE cells at the margin of the unclosed OF. However, the ectopic RPE fate is only restricted at the margin of the unclosed $\mathrm{OF}$, while the inner retinal progenitor cells in the temporal OCs in Fgfr-DCK eyes, which are mutant for FGF receptors, still adopt the NR fate. Interestingly, these FGF receptor-mutant NR progenitor cells fail to express the RGC fate determination transcription factors, Math5 and $B r n 3 b$, indicating they fail to initiate RGC development. FGF signaling has been shown to promote the initiation of RGC development in fish and chicken [31,75], and our study provides the first genetic evidence that FGF signaling also promotes the initiation of RGC development in mammals (Figure 7H). Shp2 is a FGF signaling pathway downstream mediator. It has been shown that when deleted from early OV stage using $R x$-Cre, Shp2 mutant retinal progenitor cells adopt RPE fate, while when deleted from the beginning of the OC stage using Six3-Cre, Shp 2 mutant retinas degenerate [32, 33]. When comparing the retinal cell fate phenotype of our $F g f r$ $D C K$ mice with that of $R x$-Cre; Shp $2^{f x / f x}$ mice and Six 3 Cre; $\operatorname{Shp} 2^{f x / f x}$ mice, it is interesting to note that $F g f r$ $D C K$ mice share phenotypes with both Shp2 conditional mutant mice, but less severe: the RPE cell fate is ectopically generated, however only restricted to unclosed OF region; the NR is generated, but degenerates when the mice age. The similarity and discrepancy of the phenotypes observed in these three conditional knockout mice suggest that FGF signaling plays different roles during different stages of the retina development: at the early stage, it promotes the NR fate over RPE fate; in established NR progenitor cells, it promotes retinogenesis; at the adult stage, it promotes the survival of mature retinal neurons. In the future, it would be interesting to dissect the molecular mechanisms whereby FGF signaling controls different aspects of retinogenesis and retinal homeostasis. 
In summary, we show that the OF closing process is accompanied by highly coordinated proliferation, cell fate switches, and shape changes of OFM progenitors, and that defective FGF signaling disrupts these cellular events. In addition, we have shown that FGF signaling regulates the expression of cell cycle regulators, cell fate determinants and actin regulators. Based on the experimental findings, we propose that FGF signaling controls the OF closure possibly by orchestrating multiple cellular events through regulation of expression of cell cycle regulators, cell fate determinants and actin regulators. Finally, we have shown that FGF signaling is also required for the maintenance of the OS and the development of the RGCs. However, it remains unclear which event is the primary driving force for the OF closure, how these cellular events are related to one another, whether FGF signaling directly regulates these cellular events and how FGF signaling regulates development of RGCs and the OS. In the future, the answers to these questions will be of great interest for understanding the molecular and cellular mechanisms underlying coloboma formation.

\section{Materials and Methods}

All animal work was performed in compliance with the protocols approved by the Institutional Animal Care and Use Committee at the Stowers Institute for Medical Research (SIMR). Six3Cre, Fgfr $1^{f x}$, and $F g f r 2^{f x}$ mice have been described previously [4850]. Z/EG mice [51] were purchased from Jackson Laboratory. Time-mated CD1 mice were provided by the Laboratory Animal Services Facility at SIMR. Noon on the day at which a vaginal plug is found is referred to as embryonic day 0.5 (E0.5).

\section{Tissue processing and histology}

For cryo-sections, embryos were fixed overnight in $4 \%$ formaldehyde, cryo-preserved with $15 \%$ then $30 \%$ sucrose, and frozen using a freezing bath (Thermo Scientific) with isopentane. For paraffin sections, embryos were fixed overnight in $4 \%$ formaldehyde, dehydrated through a series of gradient ethanol, and embedded in paraffin. Because the $\mathrm{OF}$ is a ventral-specific structure of the $\mathrm{OC}$, most specimens were sectioned parasagittally. The nasal-temporal orientation of the $\mathrm{OC}$ was determined based on the brain structures around the eye according to The Internet Atlas of Mouse Development [93].

mRNA in situ hybridization was performed on cryo-sections using digoxigenin-labeled RNA probes as follow: after rehydration, slides were post-fixed in $4 \%$ formaldehyde for $10 \mathrm{~min}$, digested with $1 \mu \mathrm{g} / \mathrm{ml}$ proteinase $\mathrm{K}$ for $5 \mathrm{~min}$, and fixed again for $5 \mathrm{~min}$. Slides were then acetylated with acetic anhydride for 10 min, hybridized with specific probes in a humid chamber at 56 ${ }^{\circ} \mathrm{C}$ overnight, washed, and incubated with alkaline phosphatase conjugated anti-digoxigenin antibody (Roche) at $4{ }^{\circ} \mathrm{C}$ overnight. Then slides were washed and color-developed by incubating with NBT and BCIP (Promega). The following probes were used: Fgfrl and Fgfr2 (T-cloning of RT-PCR products from E14.5 mouse embryonic head cDNA library into pGEM-T easy vector (Promega)),
Math5 and Brn3b (kindly provided by Dr Lin Gan) Shh (kindly provided by Dr Andrew McMahon), Dct (Open biosystem), and Vax2 (ATCC). Images were taken under either a Zeiss axioplan or Leica DM5500 microscope.

Immuno-histology was performed on either paraffin sections or cryo-sections. Before applying primary antibody, antigen retrieval was performed by heating slides in citrate buffer $\left(\mathrm{pH} \mathrm{6.0)}\right.$ at $95^{\circ} \mathrm{C}$ for $10 \mathrm{~min}$ then cooling at room temperature for 20 more min. After antigen retrieval, the slides were incubated with primary antibodies overnight at $4{ }^{\circ} \mathrm{C}$, washed and incubated with Alexa 488- or Alexa 568-conjugated goat or donkey secondary antibodies (Invitrogen) for 2 hours at room temperature. Slides were then washed, counter-stained with DAPI for $5 \mathrm{~min}$, washed again, and mounted using VECTASHIELD mounting medium (Vector Laboratories). The following antibodies were used: rabbit anti-Pax2 (Invitrogen), mouse anti-Pax6 (Developmental Studies Hybridoma Bank), sheep anti-Vsx2 (Chemicon), mouse anti-Mitf (lab Vision), rabbit anti-GFP (Invitrogen), goat anti-pMLC (Santa Cruz), rabbit anti-N-cadherin (Santa Cruz), rabbit anti-Pitx2 (Capra Science), Rabbit ant-Tuj1 (Convance), and mouse anti-Laminin (Sigma). The Fgfr2 (Santa Cruz) signal was amplified using a TSA kit (Perkin Elmer). Filamentous actin staining was performed by incubating slides with Alexa 488-conjugated phalloidin (Invitrogen) at room temperature for $30 \mathrm{~min}$. Images were taken under a Leica SP2 or SP5 confocal microscope.

\section{BrdU incorporation assay}

The time-mated mice were injected intraperitoneally with BrdU at $0.1 \mathrm{mg} / \mathrm{g}$ body weight two hours before sacrifice. Embryos were fixed, paraffin embedded, sectioned and immuostained for BrdU (Amersham) as described above. For each parasagittal section of the OC, about 60-100 NR progenitor cells and 20 RPE progenitor cells (based on DAPI staining) in the central region of nasal and temporal retinas and retinal cells within a 5-cell diameter from the margin of the OF were counted. For each genotype, three eyes from three mice were counted. For statistical analysis, Student's $t$-test was applied.

\section{Microdissection, microarrays and $q P C R$}

Parasagittal sections $(15 \mu \mathrm{m})$ of fresh E11.5 heads embedded in OCT were cut in a Leica cryostat, fixed in $75 \%$ ethanol for $2 \mathrm{~min}$, stained with hematoxylin for $2 \mathrm{~min}$, dehydrated with increasing ethanol series, and air-dried for $30 \mathrm{~min}$. Subsequently, retinal progenitor cells in the OF region and NR progenitor cells in the nasal and temporal quadrants from control and Fgfrl/ Fgfr2 double conditional knockout OCs were dissected using a PALM laser-microdissection microscope (Zeiss). Samples from four embryos of the same litter were pooled together to obtain sufficient materials. Total RNAs were isolated using TRIZOL (Invitrogen) and amplified twice using a T7 transcription based amplification strategy [94]. For microarray analysis, three independent RNA samples from E11.5 control and $F g f r$-DCK eyes were used. $20 \mu \mathrm{g}$ labeled cRNAs for each sample were fragmented and hybridized to Affymetrix mouse genome 4302.0 arrays. Microarray results were analyzed based on the published methods [95]. qPCR was performed on an Applied Biosystem 7900 real-time PCR machine using Power SYBR green PCR Master Mix (Applied Biosystems). For each primer pair for each experimental group, three experimental replicates and three biological replicates were performed. 
For each qPCR run, two of the following three internal controls were used: Gapdh, B2m and Atp5b. Relative expression differences between control and mutant samples and statistical analysis were analyzed using REST software [96].

\section{Bioinformatics analysis}

The LIMMA package was used to compare the gene expression changes between mutant and control samples [97]. BenjaminiYekutiele multi-test correction method was applied to control the False Discovery Rate [98]. To search for the putative binding targets for Ets 1 and Pea3, sequences 5 kilobase upstream and downstream of transcription start sites of all the differentially expressed genes were tested for matches against binding motifs for the two transcription factors in BIOBASE Database using the database's minimum-false-positive criteria. The following consensus binding matrixes are used: Pea3_Q6 (BIOBASE accession number: M00655); Ets_Q6 (BIOBASE accession number M00971).

\section{Acknowledgments}

We would like to thank D Ornitz for the Fgfr2 conditional allele, B Sandersan for help in qPCR experiments, R Krumlauf, W Neaves, M Lewallen and C Tanzie for comments, the Xie laboratory members for stimulating discussions, the Laboratory Animal Services Facility at SIMR for maintaining our mouse strains, and C Flournoy for administrative assistance. This work was supported by SIMR (T Xie), the MR and Evelyn Hudson Foundation (T Xie), and NIH grant (EY012128, Y Furuta).

\section{References}

1 Chang L, Blain D, Bertuzzi S, Brooks BP. Uveal coloboma: clinical and basic science update. Curr Opin Ophthalmol 2006; 17:447-470.

2 Uemonsa T, Sakagami K, Yasuda K, Araki M. Development of dorsal-ventral polarity in the optic vesicle and its presumptive role in eye morphogenesis as shown by embryonic transplantation and in ovo explant culturing. Dev Biol 2002; 248:319-330

3 Morcillo J, Martinez-Morales JR, Trousse F, Fermin Y, Sowden JC, Bovolenta P. Proper patterning of the optic fissure requires the sequential activity of BMP7 and SHH. Development 2006; 133:3179-3190.

4 Hero I. Optic fissure closure in the normal cinnamon mouse. An ultrastructural study. Invest Ophthalmol Vis Sci 1990; 31:197-216.

5 Favor J, Sandulache R, Neuhauser-Klaus A, et al. The mouse Pax2(1Neu) mutation is identical to a human PAX2 mutation in a family with renal-coloboma syndrome and results in developmental defects of the brain, ear, eye, and kidney. Proc Natl Acad Sci USA 1996; 93:13870-13875.

6 Otteson DC, Shelden E, Jones JM, Kameoka J, Hitchcock PF. Pax2 expression and retinal morphogenesis in the normal and Krd mouse. Dev Biol 1998; 193:209-224.

7 Torres M, Gomez-Pardo E, Gruss P. Pax2 contributes to inner ear patterning and optic nerve trajectory. Development 1996; 122:3381-3391.

8 Hallonet M, Hollemann T, Pieler T, Gruss P. Vax1, a novel homeobox-containing gene, directs development of the basal forebrain and visual system. Genes Dev 1999; 13:3106-3114.

9 Mui SH, Kim JW, Lemke G, Bertuzzi S. Vax genes ventralize the embryonic eye. Genes Dev 2005; 19:1249-1259.

10 Gage PJ, Suh H, Camper SA. Dosage requirement of Pitx2 for development of multiple organs. Development 1999; 126:4643-4651.

11 Huh S, Hatini V, Marcus RC, Li SC, Lai E. Dorsal-ventral patterning defects in the eye of BF-1-deficient mice associated with a restricted loss of shh expression. Dev Biol 1999; 211:53-63.

12 Weston CR, Wong A, Hall JP, Goad ME, Flavell RA, Davis RJ. JNK initiates a cytokine cascade that causes Pax2 expression and closure of the optic fissure. Genes Dev 2003; 17:1271-1280.

13 Kastner P, Grondona JM, Mark M, et al. Genetic analysis of RXR alpha developmental function: convergence of RXR and RAR signaling pathways in heart and eye morphogenesis. Cell 1994; 78:987-1003.

14 Matt N, Dupe V, Garnier JM, et al. Retinoic acid-dependent eye morphogenesis is orchestrated by neural crest cells. Development 2005; 132:4789-4800.

15 Zhou CJ, Molotkov A, Song L, et al. Ocular coloboma and dorsoventral neuroretinal patterning defects in Lrp6 mutant eyes. Dev Dyn 2008; 237:3681-3689.

16 Liu C, Nathans J. An essential role for frizzled 5 in mammalian ocular development. Development 2008; 135:3567-3576.

17 Kim TH, Goodman J, Anderson KV, Niswander L. Phactr4 regulates neural tube and optic fissure closure by controlling PP1-, Rb-, and E2F1-regulated cell-cycle progression. Dev Cell 2007; 13:87-102.

18 Pirity MK, Wang WL, Wolf LV, Tamm ER, Schreiber-Agus N, Cvekl A. Rybp, a Polycomb complex-associated protein, is required for mouse eye development. BMC Dev Biol 2007; 7:39.

19 Cayouette M, Poggi L, Harris WA. Lineage in the vertebrate retina. Trends Neurosci 2006; 29:563-570.

20 Cepko CL, Austin CP, Yang X, Alexiades M, Ezzeddine D. Cell fate determination in the vertebrate retina. Proc Natl Acad Sci USA 1996; 93:589-595.

21 Yang Z, Ding K, Pan L, Deng M, Gan L. Math5 determines the competence state of retinal ganglion cell progenitors. Dev Biol 2003; 264:240-254.

22 Kim J, Wu HH, Lander AD, Lyons KM, Matzuk MM, Calof AL. GDF11 controls the timing of progenitor cell competence in developing retina. Science 2005; 308:1927-1930.

23 Zhang XM, Yang XJ. Regulation of retinal ganglion cell production by Sonic hedgehog. Development 2001; 128:943-957.

24 Mason I. Initiation to end point: the multiple roles of fibroblast growth factors in neural development. Nat Rev Neurosci 2007; 8:583-596.

25 Thisse B, Thisse C. Functions and regulations of fibroblast growth factor signaling during embryonic development. Dev Biol 2005; 287:390-402.

26 Moore KB, Mood K, Daar IO, Moody SA. Morphogenetic movements underlying eye field formation require interactions between the FGF and ephrinB1 signaling pathways. Dev Cell 2004; 6:55-67.

27 Hyer J, Mima T, Mikawa T. FGF1 patterns the optic vesicle by directing the placement of the neural retina domain. Devel- 
opment 1998; 125:869-877.

28 Pittack C, Grunwald GB, Reh TA. Fibroblast growth factors are necessary for neural retina but not pigmented epithelium differentiation in chick embryos. Development 1997; 124:805816.

29 Picker A, Brand M. Fgf signals from a novel signaling center determine axial patterning of the prospective neural retina. Development 2005; 132:4951-4962.

30 Guillemot F, Cepko CL. Retinal fate and ganglion cell differentiation are potentiated by acidic FGF in an in vitro assay of early retinal development. Development 1992; 114:743-754.

31 Martinez-Morales JR, Del Bene F, Nica G, Hammerschmidt M, Bovolenta P, Wittbrodt J. Differentiation of the vertebrate retina is coordinated by an FGF signaling center. Dev Cell 2005; 8:565-574.

32 Cai Z, Feng GS, Zhang X. Temporal requirement of the protein tyrosine phosphatase Shp2 in establishing the neuronal fate in early retinal development. J Neurosci 2010; 30:41104119.

33 Cai Z, Simons DL, Fu XY, Feng GS, Wu SM, Zhang X. Loss of Shp2-mediated mitogen-activated protein kinase signaling in Muller glial cells results in retinal degeneration. Mol Cell Biol 2011; 31:2973-2983.

34 Itoh N, Ornitz DM. Functional evolutionary history of the mouse Fgf gene family. Dev Dyn 2008; 237:18-27.

35 Nguyen M, Arnheiter H. Signaling and transcriptional regulation in early mammalian eye development: a link between FGF and MITF. Development 2000; 127:3581-3591.

36 Dono R, Texido G, Dussel R, Ehmke H, Zeller R. Impaired cerebral cortex development and blood pressure regulation in FGF-2-deficient mice. EMBO J 1998; 17:4213-4225.

37 Miller DL, Ortega S, Bashayan O, Basch R, Basilico C. Compensation by fibroblast growth factor 1 (FGF1) does not account for the mild phenotypic defects observed in FGF2 null mice. Mol Cell Biol 2000; 20:2260-2268.

38 Ortega S, Ittmann M, Tsang SH, Ehrlich M, Basilico C. Neuronal defects and delayed wound healing in mice lacking fibroblast growth factor 2. Proc Natl Acad Sci USA 1998; 95:5672-5677.

39 Colvin JS, Green RP, Schmahl J, Capel B, Ornitz DM. Maleto-female sex reversal in mice lacking fibroblast growth factor 9. Cell 2001; 104:875-889.

40 Zhao S, Hung FC, Colvin JS, et al. Patterning the optic neuroepithelium by FGF signaling and Ras activation. Development 2001; 128:5051-5060.

41 Sun X, Meyers EN, Lewandoski M, Martin GR. Targeted disruption of Fgf8 causes failure of cell migration in the gastrulating mouse embryo. Genes Dev 1999; 13:1834-1846.

42 Colvin JS, Bohne BA, Harding GW, McEwen DG, Ornitz DM. Skeletal overgrowth and deafness in mice lacking fibroblast growth factor receptor 3. Nat Genet 1996; 12:390-397.

43 Deng C, Wynshaw-Boris A, Zhou F, Kuo A, Leder P. Fibroblast growth factor receptor 3 is a negative regulator of bone growth. Cell 1996; 84:911-921.

44 Weinstein M, Xu X, Ohyama K, Deng CX. FGFR-3 and FGFR-4 function cooperatively to direct alveogenesis in the murine lung. Development 1998; 125:3615-3623.

45 Arman E, Haffner-Krausz R, Chen Y, Heath JK, Lonai P. Targeted disruption of fibroblast growth factor (FGF) receptor
2 suggests a role for FGF signaling in pregastrulation mammalian development. Proc Natl Acad Sci USA 1998; 95:50825087.

46 Deng CX, Wynshaw-Boris A, Shen MM, Daugherty C, Ornitz DM, Leder P. Murine FGFR-1 is required for early postimplantation growth and axial organization. Genes Dev 1994; 8:3045-3057.

47 Yamaguchi TP, Harpal K, Henkemeyer M, Rossant J. fgfr-1 is required for embryonic growth and mesodermal patterning during mouse gastrulation. Genes Dev 1994; 8:3032-3044.

48 Furuta Y, Lagutin O, Hogan BL, Oliver GC. Retina- and ventral forebrain-specific Cre recombinase activity in transgenic mice. Genesis 2000; 26:130-132.

49 Xu X, Qiao W, Li C, Deng CX. Generation of Fgfr1 conditional knockout mice. Genesis 2002; 32:85-86.

$50 \mathrm{Yu} \mathrm{K}, \mathrm{Xu} \mathrm{J}$, Liu Z, et al. Conditional inactivation of FGF receptor 2 reveals an essential role for FGF signaling in the regulation of osteoblast function and bone growth. Development 2003; 130:3063-3074.

51 Novak A, Guo C, Yang W, Nagy A, Lobe CG. Z/EG, a double reporter mouse line that expresses enhanced green fluorescent protein upon Cre-mediated excision. Genesis 2000; 28:147155.

52 Turner N, Grose R. Fibroblast growth factor signalling: from development to cancer. Nat Rev Cancer 2010; 10:116-129.

53 Barbieri AM, Lupo G, Bulfone A, et al. A homeobox gene, vax2, controls the patterning of the eye dorsoventral axis. Proc Natl Acad Sci USA 1999; 96:10729-10734.

54 Barbieri AM, Broccoli V, Bovolenta $\mathrm{P}$, et al. Vax2 inactivation in mouse determines alteration of the eye dorsal-ventral axis, misrouting of the optic fibres and eye coloboma. Development 2002; 129:805-813.

55 Mic FA, Molotkov A, Fan X, Cuenca AE, Duester G. RALDH3, a retinaldehyde dehydrogenase that generates retinoic acid, is expressed in the ventral retina, otic vesicle and olfactory pit during mouse development. Mech Dev 2000; 97:227-230.

56 Ekker SC, Ungar AR, Greenstein P, et al. Patterning activities of vertebrate hedgehog proteins in the developing eye and brain. Curr Biol 1995; 5:944-955.

57 Macdonald R, Barth KA, Xu Q, Holder N, Mikkola I, Wilson SW. Midline signalling is required for Pax gene regulation and patterning of the eyes. Development 1995; 121:32673278.

58 Schwarz M, Cecconi F, Bernier G, et al. Spatial specification of mammalian eye territories by reciprocal transcriptional repression of Pax2 and Pax6. Development 2000; 127:43254334.

59 Baumer N, Marquardt T, Stoykova A, et al. Retinal pigmented epithelium determination requires the redundant activities of Pax2 and Pax6. Development 2003; 130:2903-2915.

60 Horsford DJ, Nguyen MT, Sellar GC, Kothary R, Arnheiter $\mathrm{H}$, McInnes RR. Chx10 repression of Mitf is required for the maintenance of mammalian neuroretinal identity. Development 2005; 132:177-187.

61 Rowan S, Chen C-MA, Young TL, Fisher DE, Cepko CL. Transdifferentiation of the retina into pigmented cells in ocular retardation mice defines a new function of the homeodomain gene Chx10 10.1242/dev.01300. Development 2004; 
131:5139-5152.

62 Hodgkinson CA, Moore KJ, Nakayama A, et al. Mutations at the mouse microphthalmia locus are associated with defects in a gene encoding a novel basic-helix-loop-helix-zipper protein. Cell 1993; 74:395-404.

63 Geeraets R. An electron microscopic study of the closure of the optic fissure in the golden hamster. Am J Anat 1976; 145:411-431.

64 Lecuit T, Lenne PF. Cell surface mechanics and the control of cell shape, tissue patterns and morphogenesis. Nat Rev Mol Cell Biol 2007; 8:633-644.

65 Quintin S, Gally C, Labouesse M. Epithelial morphogenesis in embryos: asymmetries, motors and brakes. Trends Genet 2008; 24:221-230.

66 Eiraku M, Takata N, Ishibashi H, et al. Self-organizing opticcup morphogenesis in three-dimensional culture. Nature 2011; 472:51-56.

67 Tam PP, Behringer RR. Mouse gastrulation: the formation of a mammalian body plan. Mech Dev 1997; 68:3-25.

68 Keller R. Mechanisms of elongation in embryogenesis. Development 2006; 133:2291-2302.

69 Wittmann T, Waterman-Storer CM. Cell motility: can Rho GTPases and microtubules point the way? J Cell Sci 2001; 114:3795-3803.

70 Geese M, Loureiro JJ, Bear JE, Wehland J, Gertler FB, Sechi AS. Contribution of Ena/VASP proteins to intracellular motility of listeria requires phosphorylation and proline-rich core but not F-actin binding or multimerization. Mol Biol Cell 2002; 13:2383-2396.

71 Iizaka M, Han HJ, Akashi H, et al. Isolation and chromosomal assignment of a novel human gene, CORO1C, homologous to coronin-like actin-binding proteins. Cytogenet Cell Genet 2000; 88:221-224.

72 Parast MM, Otey CA. Characterization of palladin, a novel protein localized to stress fibers and cell adhesions. $J$ Cell Biol 2000; 150:643-656.

73 Sato A, Khadka DK, Liu W, et al. Profilin is an effector for Daam1 in non-canonical Wnt signaling and is required for vertebrate gastrulation. Development 2006; 133:4219-4231.

74 Brent AE, Tabin CJ. FGF acts directly on the somitic tendon progenitors through the Ets transcription factors Pea3 and Erm to regulate scleraxis expression. Development 2004; 131:3885-3896.

75 McCabe KL, Gunther EC, Reh TA. The development of the pattern of retinal ganglion cells in the chick retina: mechanisms that control differentiation. Development 1999; 126:5713-5724.

76 Pan L, Deng M, Xie X, Gan L. ISL1 and BRN3B co-regulate the differentiation of murine retinal ganglion cells. Development 2008; 135:1981-1990.

77 Baumer N, Marquardt T, Stoykova A, Ashery-Padan R, Chowdhury K, Gruss P. Pax6 is required for establishing naso-temporal and dorsal characteristics of the optic vesicle. Development 2002; 129:4535-4545.

78 Sanyanusin P, Schimmenti LA, McNoe LA, et al. Mutation of the PAX2 gene in a family with optic nerve colobomas, renal anomalies and vesicoureteral reflux. Nat Genet 1995; 9:358364.

79 Masai I, Lele Z, Yamaguchi M, et al. N-cadherin mediates re- tinal lamination, maintenance of forebrain compartments and patterning of retinal neurites. Development 2003; 130:24792494.

80 Take-uchi M, Clarke JD, Wilson SW. Hedgehog signalling maintains the optic stalk-retinal interface through the regulation of Vax gene activity. Development 2003; 130:955-968.

81 Roffers-Agarwal J, Xanthos JB, Kragtorp KA, Miller JR. Enabled (Xena) regulates neural plate morphogenesis, apical constriction, and cellular adhesion required for neural tube closure in Xenopus. Dev Biol 2008; 314:393-403.

82 Ding Z, Lambrechts A, Parepally M, Roy P. Silencing profilin-1 inhibits endothelial cell proliferation, migration and cord morphogenesis. J Cell Sci 2006; 119:4127-4137.

83 Moldovan NI, Milliken EE, Irani K, et al. Regulation of endothelial cell adhesion by profilin. Curr Biol 1997; 7:24-30.

84 Ridley AJ. Rho GTPases and actin dynamics in membrane protrusions and vesicle trafficking. Trends Cell Biol 2006; 16:522-529.

85 Gryzik T, Muller HA. FGF8-like1 and FGF8-like2 encode putative ligands of the FGF receptor $\mathrm{Htl}$ and are required for mesoderm migration in the Drosophila gastrula. Curr Biol 2004; 14:659-667.

86 Stathopoulos A, Tam B, Ronshaugen M, Frasch M, Levine M. pyramus and thisbe: FGF genes that pattern the mesoderm of Drosophila embryos. Genes Dev 2004; 18:687-699.

87 Barrett K, Leptin M, Settleman J. The Rho GTPase and a putative RhoGEF mediate a signaling pathway for the cell shape changes in Drosophila gastrulation. Cell 1997; 91:905-915.

88 Smallhorn M, Murray MJ, Saint R. The epithelial-mesenchymal transition of the Drosophila mesoderm requires the Rho GTP exchange factor Pebble. Development 2004; 131:26412651.

89 Kolsch V, Seher T, Fernandez-Ballester GJ, Serrano L, Leptin M. Control of Drosophila gastrulation by apical localization of adherens junctions and RhoGEF2. Science 2007; 315:384386.

90 Nikolaidou KK, Barrett K. A Rho GTPase signaling pathway is used reiteratively in epithelial folding and potentially selects the outcome of Rho activation. Curr Biol 2004; 14:18221826.

91 Dorey K, Amaya E. FGF signalling: diverse roles during early vertebrate embryogenesis. Development 2010; 137:37313742.

92 Hyer J, Mima T, Mikawa T. FGF1 patterns the optic vesicle by directing the placement of the neural retina domain. Development 1998; 125:869-877.

93 Williams BS, Doyle MD. An Internet atlas of mouse development. Comput Med Imaging Graph 1996; 20:433-447.

94 Van Gelder RN, von Zastrow ME, Yool A, Dement WC, Barchas JD, Eberwine JH. Amplified RNA synthesized from limited quantities of heterogeneous cDNA. Proc Natl Acad Sci USA 1990; 87:1663-1667.

95 Yoshida S, Mears AJ, Friedman JS, et al. Expression profiling of the developing and mature $\mathrm{Nrl}^{-/-}$mouse retina: identification of retinal disease candidates and transcriptional regulatory targets of Nrl. Hum Mol Genet 2004; 13:1487-1503.

96 Pfaffl MW, Horgan GW, Dempfle L. Relative expression software tool (REST) for group-wise comparison and statistical analysis of relative expression results in real-time PCR. 
Nucleic Acids Res 2002; 30:e36.

97 Smyth GK. Linear models and empirical bayes methods for assessing differential expression in microarray experiments. Stat Appl Genet Mol Biol 2004; 3:Article3.
98 Benjamini Y, Yekutieli D. The control of the false discovery rate in multiple testing under dependency. Annals of Statistics 2001; 29:1165-1188.

(Supplementary information is linked to the online version of the paper on the Cell Research website.) 